\title{
Slave rotor approach to dynamically screened Coulomb interactions in solids
}

\author{
I. S. Krivenko ${ }^{1,2}$ and S. Biermann ${ }^{1}$ \\ ${ }^{1}$ Centre de Physique Théorique, Ecole Polytechnique, \\ CNRS UMR7644, 91128 Palaiseau Cedex, France \\ ${ }^{2}$ I. Institut für Theoretische Physik, Universität Hamburg, Jungiusstraße 9, 20355 Hamburg, Germany
}

(Dated: June 18, 2021)

\begin{abstract}
Recent studies of dynamical screening of the electronic Coulomb interactions in solids have revived interest in lattice models of correlated fermions coupled to bosonic degrees of freedom (HubbardHolstein-type models). We propose a dynamical mean-field-based approach to dynamically screened Coulomb interactions. In the effective Anderson-Holstein model, a transformation to slave rotors [S. Florens and A. Georges, Phys. Rev. B 66165111 (2002)] is performed to decouple the dynamical part of the interaction. This transformation allows for a systematic derivation and analysis of recently introduced approximate schemes for the solution of dynamical impurity problems, in particular, the Bose factor ansatz within the dynamic atomic limit approximation (DALA) with and without Lang-Firsov correction. More importantly still, it suggests an optimized choice for a Bose factor in the sense of the variational principle of Feynman and Peierls. We demonstrate the accuracy of our scheme and present a comparison to calculations within the DALA.

PACS numbers: 71.27.+a, 71.45.Gm, 71.10.-w
\end{abstract}

\section{INTRODUCTION}

Lattice models of correlated fermions coupled to collective bosonic modes have attracted renewed interest recently. The Hubbard-Holstein mode $\mathrm{I}^{11}$ is a paradigmatic model to study the interplay between phonon-mediated attraction of electrons and their electrostatic Coulomb repulsion ${ }^{2}$. The model exhibits a wide variety of phenomena, such as bipolaron formation ${ }^{3 / 4}$, charge density waves $(\mathrm{CDW})$ and phonon-induced superconductivity $\sqrt{516}$, a metal-insulator (Mott) transition affected by the phonons ${ }^{788}$, and non-Fermi-liquid behavior ${ }^{9}$.

Besides the more traditional context of electronphonon coupling, the Hubbard-Holstein model (or, equivalently, the Hubbard model with frequency-dependent interactions) has been acquiring new applications within realistic theories of correlated materials10111. Techniques based on density functional theory (DFT) are used to compute the one-particle band structure of a material as a first step of combined numerical schemes such as local-density approximation plus dynamical mean-field theory $(\mathrm{LDA}+\mathrm{DMFT})^{12113}$ and LDA $+U^{144}$. On the next step, electrons from a subset of correlated bands are identified with fermionic degrees of freedom of a lattice model. This downfolding procedure allows one to subsequently apply established many-body methods (in particular, DMFT) ${ }^{15}$ ) to account for correlation effects and obtain electronic spectral functions 16/17, $^{16}$ optical conductivities $^{18 \mid 19}$, or transport properties (see, e.g., 20).

This kind of calculation has recently acquired a new level of realism thanks to techniques allowing for firstprinciples calculations of the effective local Hubbard interactions also: The constrained random-phase approximation (cRPA) ${ }^{2123}$ even gives access to the energydependent matrix elements of the interaction. The energy dependence of this Hubbard interaction $\mathcal{U}(\omega)$ reflects the fact that the high-energy itinerant states, which are projected out from the full band structure, dynamically screen the Coulomb interactions between correlated electrons. These screening processes quite generally result in a substantial decrease of the static density-density interaction as parametrized by an energydependent Slater parameter $F_{0}(\omega)$. They furthermore lead to renormalizations of the one-particle hopping 24 . As a result, the phase diagram of the lattice model is substantially affected.

Dynamical impurity models are also a central ingredient of the GW+DMFT method ${ }^{25 / 26}$, combining manybody perturbation theory in the framework of Hedin's GW approximation with DMFT. Indeed, inspired by extended DMFT27, this method maps a system with long-range interactions onto an effective local problem with dynamical interactions, subject to a double self-consistency condition relating one- and two-particle propagators to their counterparts in the solid. The need for solving this dynamical effective impurity problem has been a serious bottleneck hindering the implementation of the scheme for quite some time, but the recent development of the Bose factor ansatz (BFA) ${ }^{11}$ as an efficient impurity solver has finally unblocked the field 2829 . For a review of the current status, sec ${ }^{30}$.

Dynamical mean-field theory applied to the HubbardHolstein model with local phonons maps it onto an effective Anderson-Holstein model, parametrized by a bath hybridization function $\Delta(i \omega)$, which is subject to a self-consistency condition. Solving the effective impurity model numerically amounts to obtaining its thermal Green's function, $G(\tau)=-\left\langle\mathbb{T} d(\tau) d^{\dagger}(0)\right\rangle$, where the average is performed by using the action corresponding to the effective Anderson-Holstein model. Inclusion of bosons into an impurity problem drastically enlarges the dimensionality of its state space, making it much more difficult to solve.

In principle, it is still possible to apply continuous- 
time quantum Monte Carlo (CTQMC) algorithms to the Anderson-Holstein model. In practice, and in particular for applications to realistic materials, this is, however, not so straightforward: The weak-coupling algorithm by Rubtsov $\sqrt{31}$ can treat interactions with arbitrary frequency dependence, provided they are not too strong (in comparison to the bandwidth) and do not contain high-frequency components. In general, neither of these conditions is satisfied for realistic screened interactions effective impurity models are usually found in antiadiabatic or intermediate regimes. A recently proposed generalization of the strong-coupling algorithm by Werner and Millis ${ }^{32}$ does not suffer from these problems, and has allowed for several recent applications within electronic structure calculations; see, e.g., 10 and 33 . Nevertheless, there is still an infamous problem associated with the extraction of real-frequency spectral data from imaginary-time results of a QMC run. A noticeable part of the spectral weight (replica of the low-energy electronic structure) can lie far outside the bare electronic band, when the impurity model is in the antiadiabatic regime. Resolution of spectra of this kind is notoriously difficult for analytic continuation tools.

Motivated by the mentioned problems, a new family of techniques has emerged over the last few years. The "Bose factor ansatz" (BFA) within the dynamic atomic limit approximation (DALA) ${ }^{11}$ was proposed as an approximate - yet accurate - scheme capable to circumvent the analytic continuation problem by reducing a given Anderson-Holstein problem to an effective Anderson model. The resulting Anderson model is readily solvable by existing impurity solvers, and the effect of the bosonic resonances is treated analytically within the BFA. Even the above-mentioned reduction of the effective bandwidth compared to its bare value ${ }^{24}$ can be taken into account: the change can be estimated by means of a Lang-Firsov transformation ${ }^{34 / 35}$ and then incorporated into the DALA approach (DALA+LF) ${ }^{11}$.

Following this route of study, in the present paper we introduce an approach to the Anderson-Holstein model based on a slave rotor representation ${ }^{36}$. Slave rotor variables were introduced in 36 and 37 as an efficient means to decouple charge and spin degrees in low-energy models for correlated materials, even in the case of dynamically screened interactions. For impurity models in the DMFT context, the formalism leads to a practical scheme allowing for an approximate solution of the DMFT equations that correctly reproduces the Mott transition and the main spectral features associated to it. In contrast to previous work, however, we use the slave rotors here to decouple the dynamical part of the density-density interaction only. It becomes clear within this framework, that DALA and DALA+LF can be understood as simple approximations made on fluctuations of the slave phase field. Finally, we derive an effective mean-field modulation of the hybridization function $\Delta(i \omega)$ induced by the coupling to the phase variables, leading to an optimized Bose factor ansatz in the sense of Feynman's variational principle.

This paper is organized as follows. In Sec. II], we formulate the Hubbard-Holstein model and an equivalent Hubbard model with energy-dependent Hubbard interactions. Within DMFT, this model is mapped onto an Anderson-Holstein model with energy-dependent $\mathcal{U}(\omega)$. In Sec. III. we analyze this model within a slave rotor transformation. Different existing approximations (DALA, DALA+LF) are found to be specific approximations to the slave rotors equations, as explained in Sec. IV] A consequence of the coupling between lattice fermions and bosonic degrees of freedom is a reduction of spectral weight in the low-energy sector of the model, rationalized as an electronic polaron effect ${ }^{24}$. In Sec. V we derive a finite-temperature generalization for the expression of the bosonic renormalization factor $Z_{B}$ and compare it to an improved estimate within the slave rotor formalism. Finally, a scheme beyond DALA and DALA+LF is proposed in Sec. VI Derived from Feynman's variational principle, this optimized Bose factor ansatz contains both the high-energy plasmon replica and the low-energy spectral weight reduction in a consistent way. Section VII contains a brief discussion of the physical meaning of the optimized Bose factor ansatz. Section VIII summarizes the resulting self-consistency loop and gives technical details. In Sec. IX we present DMFT results for the Hubbard-Holstein model obtained using our slave rotor scheme. Finally, Sec. $\mathrm{X}$ concludes the paper. Two appendices present additional details concerning the derivation of higher-order correlation functions within the slave rotor picture, as well as of a temperaturedependent Lang-Firsov factor.

\section{MODELS WITH SCREENED COULOMB INTERACTION}

In the present paper, we will focus on the single-band Hubbard model with dynamically screened Coulomb interactions $\mathcal{U}(\omega)$. Electrons can hop from site to site on a periodic lattice: mathematically, electrons of spin $\sigma$ are created (annihilated) on site $i$ by operators $d_{i \sigma}^{\dagger}\left(d_{i \sigma}\right)$. The model is defined by the finite-temperature action,

$$
\begin{aligned}
S_{H}= & -\sum_{i j, \sigma} \int_{0}^{\beta} d \tau \bar{d}_{i \sigma}(\tau)\left[\left(-\partial_{\tau}+\mu\right) \delta_{i j}+t_{i j}\right] d_{j \sigma}(\tau)+ \\
& +U_{\infty} \sum_{i} \int_{0}^{\beta} d \tau n_{i \uparrow}(\tau) n_{i \downarrow}(\tau)+ \\
+ & \frac{1}{2} \sum_{i} \iint_{0}^{\beta} d \tau d \tau^{\prime} N_{i}(\tau) U_{\text {ret }}\left(\tau-\tau^{\prime}\right) N_{i}\left(\tau^{\prime}\right) .
\end{aligned}
$$

Here, $t_{i j}$ are hopping amplitudes between adjacent atoms on the lattice and $\mu$ is the chemical potential. The full density operators are defined as $N_{i}=n_{i \uparrow}+n_{i \downarrow}$. The instantaneous part of the interaction is denoted by $U_{\infty}$. Screening is contained in the retarded part $U_{\text {ret }}(i \nu)$, 
which is chosen to be negative and thus effectively reduces the on-site electron-electron repulsion.

The retarded interaction $U_{\text {ret }}(\tau)$ can be represented as a superposition of modes each parametrized by a position of a resonance $\omega_{\alpha}$ and coupling strength $\lambda_{\alpha}^{2}$ :

$$
U_{\text {ret }}(\tau)=-\sum_{\alpha} \lambda_{\alpha}^{2} \frac{\cosh \left[(\tau-\beta / 2) \omega_{\alpha}\right]}{\sinh \left[\omega_{\alpha} \beta / 2\right]} .
$$

This expression is valid for $\tau \in[0 ; \beta)$ and must be periodically continued outside the segment. We will also need a Matsubara frequency variant of this expansion,

$$
U_{\text {ret }}(i \nu)=-\sum_{\alpha} \lambda_{\alpha}^{2} \frac{2 \omega_{\alpha}}{\nu^{2}+\omega_{\alpha}^{2}}
$$

with bosonic Matsubara frequencies $\nu=\nu_{n}=n \frac{2 \pi}{\beta}$, as well as an equivalent real-frequency description,

$$
U_{\text {ret }}(\tau)=-\int_{0}^{+\infty} \Im U_{\text {ret }}(\epsilon) \frac{\cosh [(\tau-\beta / 2) \epsilon]}{\sinh [\epsilon \beta / 2]} \frac{d \epsilon}{\pi},
$$

which uses a screening spectral function $\Im U_{\text {ret }}(\epsilon)=$ $-\pi \lambda^{2}(\epsilon), \lambda(\epsilon)=\sum_{\alpha} \lambda_{\alpha}^{2}\left[\delta\left(\epsilon-\omega_{\alpha}\right)-\delta\left(\epsilon+\omega_{\alpha}\right)\right]$.

The $\tau$ dependence of the interaction makes it necessary to use the path-integral formalism and action (1) instead of a Hamiltonian. However, in some cases, it is more convenient to introduce a set of bosonic modes at each lattice site with frequencies $\omega_{\alpha}$ and write a HubbardHolstein model Hamiltonian, which is equivalent to $S_{H}$. In this case, the frequencies $\omega_{\alpha}$ are identified with plasmonic resonances (the "charge cloud" of the integrated out electrons plays the role of the plasma). The Hamiltonian includes a term which couples electrons to the introduced bosons,

$$
\begin{aligned}
& \hat{H}_{\mathrm{HH}}= \\
& -\sum_{i j, \sigma} t_{i j} d_{i \sigma}^{\dagger} d_{j \sigma}-\mu \sum_{i, \sigma} d_{i \sigma}^{\dagger} d_{i \sigma}+U_{\infty} \sum_{i} d_{i \uparrow}^{\dagger} d_{i \uparrow} d_{i \downarrow}^{\dagger} d_{i \downarrow}+ \\
& \quad+\sum_{i, \alpha} \omega_{\alpha} b_{i \alpha}^{\dagger} b_{i \alpha}+\sum_{i, \alpha, \sigma} \lambda_{\alpha} d_{i \sigma}^{\dagger} d_{i \sigma}\left(b_{i \alpha}^{\dagger}+b_{i \alpha}\right) .
\end{aligned}
$$

The equivalence of $S_{H}$ and $\hat{H}_{\mathrm{HH}}$ is readily verified by integrating out the $b_{i \alpha}^{\dagger}, b_{i \alpha}$ variables.

It is worth noting that the Hubbard-Holstein model and the corresponding action $S_{H}$ may be supplemented with additional site-local electron terms (for instance, a local magnetic field), and most of the results of the present paper will stand.

\section{SLAVE ROTOR TRANSFORMATION OF THE ANDERSON-HOLSTEIN MODEL}

The slave rotor approach invented by Florens and Georges $^{36}$ is an elegant and economic way to separate out and describe charge fluctuation in models of strongly correlated electrons. It was successfully applied to both impurity ${ }^{37}$ and lattice models $\frac{38139}{\text {, to study }}$ the bandwidth-controlled and doping-controlled Mott transition 40, as well as to magnetism of multiorbital model $\$ 41$. Among other results, a slave rotor decoupling of the screened Coulomb interaction $U(\tau)$ was described by Florens in [37. Here, we choose a different form of such a decoupling and provide a short reasoning for the choice later in this section.

Within the slave rotor picture, one introduces a "rotor phase" variable $\theta$, which is conjugate to the full charge, and a new pair of fermionic variables $\bar{f}_{\sigma}, f_{\sigma}$ (called spinons hereafter). The rotor phase variable is related to the Hubbard-Stratonovich scalar $\phi$ field, which is often used to decouple density-density interactions between electrons,

$$
\phi(\tau) \equiv \frac{\partial \theta}{\partial \tau}, \quad \theta(\tau) \in[0 ; 2 \pi), \quad \theta(0)=\theta(\beta),
$$

where the new fermion variables are proportional to the old ones with an additional complex phase given by $\theta$,

$$
f_{\sigma} \equiv d_{\sigma} e^{i \theta}, \quad \bar{f}_{\sigma} \equiv \bar{d}_{\sigma} e^{-i \theta} .
$$

The switch of variables $\phi \mapsto \theta$ and $\bar{d}, d \mapsto \bar{f}, f$ is linear and thus the corresponding Jacobians of path integrals are irrelevant constants. It is also worth noting that $\theta$ is introduced in such a way that there is no need to consider its static component: the resulting action and any correlation function appearing in the theory contain either a $\tau$ derivative of $\theta$ or a difference $\theta(\tau)-\theta\left(\tau^{\prime}\right)$.

The thermal Green's function of the original electrons is readily expressed in terms of the new variables using definition (7),

$$
G(\tau) \equiv-\left\langle d_{\sigma}(\tau) \bar{d}_{\sigma}(0)\right\rangle=-\left\langle f_{\sigma}(\tau) \bar{f}_{\sigma}(0) e^{-i \theta(\tau)+i \theta(0)}\right\rangle .
$$

When the slave rotor transformation is used to study lattice models, the degrees of freedom are usually introduced separately at each lattice site. Some sort of a mean-field (saddle-point) approximation is then applied to the phase variables $\theta_{i}$. This procedure allows for a decoupling of spinons and chargons and to estimate the role of either of the two subsystems in a given physical phenomenon.

Here, we use the slave rotor representation in a slightly different fashion. Our approach is based on dynamical mean-field theory 7115 (DMFT), mapping the lattice (Hubbard-Holstein) model onto an effective Anderson impurity model with frequency-dependent interactions $U(i \nu)$. This single-site Anderson model is parametrized by a hybridization function $\Delta(i \omega)$, which is to be determined self-consistently. The self-consistency condition is dictated by the bare electronic band structure of the lattice or crystal, i.e., by hopping matrix elements $t_{i j}$ (see Sec. VII for more details). The action of the auxiliary Anderson model reads

$$
S_{\mathrm{AM}}=S_{\mathrm{AM}}^{\mathrm{st}}+S_{\mathrm{AM}}^{\mathrm{dyn}},
$$




$$
\begin{aligned}
& S_{\mathrm{AM}}^{\mathrm{st}}=-\sum_{\sigma} \int_{0}^{\beta} d \tau \bar{d}_{\sigma}(\tau)\left[-\partial_{\tau}+\tilde{\mu}\right] d_{\sigma}(\tau)+ \\
&++\sum_{\sigma} \iint_{0}^{\beta} d \tau d \tau^{\prime} \bar{d}_{\sigma}(\tau) \Delta\left(\tau-\tau^{\prime}\right) d_{\sigma}\left(\tau^{\prime}\right)+ \\
&+U_{0} \int_{0}^{\beta} d \tau n_{\uparrow}(\tau) n_{\downarrow}(\tau), \\
& S_{\mathrm{AM}}^{\mathrm{dyn}}=\frac{1}{2} \iint_{0}^{\beta} d \tau d \tau^{\prime} N(\tau) \bar{U}\left(\tau-\tau^{\prime}\right) N\left(\tau^{\prime}\right) .
\end{aligned}
$$

In the original action (1) of the Hubbard model the full interaction function is split into an unscreened part $U_{\infty}$ and a retarded part: $U(\tau)=U_{\infty} \delta(\tau)+U_{\text {ret }}(\tau)$. Here, in contrast, we have explicitly extracted the fully screened static component: $U(\tau)=U_{0} \delta(\tau)+\bar{U}(\tau)$, where $U_{0} \equiv U(i \nu=0)=U_{\infty}-2 \sum_{\alpha} \lambda_{\alpha}^{2} / \omega_{\alpha}$ (and the chemical potential has also undergone a modification, $\left.\tilde{\mu}=\mu+\sum_{\alpha} \lambda_{\alpha}^{2} / \omega_{\alpha}\right)$. This has been done to proceed with a Hubbard-Stratonovich decoupling of $S_{\mathrm{AM}}^{\mathrm{dyn}}$ alone:

$$
\begin{aligned}
& S_{\mathrm{AM}}=-\sum_{\sigma} \int_{0}^{\beta} d \tau \bar{d}_{\sigma}(\tau)\left[-\partial_{\tau}+\tilde{\mu}-i \phi(\tau)\right] d_{\sigma}(\tau)+ \\
&+\sum_{\sigma} \iint_{0}^{\beta} d \tau d \tau^{\prime} \bar{d}_{\sigma}(\tau) \Delta\left(\tau-\tau^{\prime}\right) d_{\sigma}\left(\tau^{\prime}\right)+ \\
&+U_{0} \int_{0}^{\beta} d \tau n_{\uparrow}(\tau) n_{\downarrow}(\tau)+S_{\phi}, \\
& S_{\phi}=\frac{1}{2} \iint_{0}^{\beta} d \tau d \tau^{\prime} \phi(\tau) \bar{U}^{-1}\left(\tau-\tau^{\prime}\right) \phi\left(\tau^{\prime}\right)
\end{aligned}
$$

Here, one sees the difference between the present approach and the approach by Florens et al. In [37], a $\phi$ field was used to decouple the full interaction term in the Anderson model, including both dynamical and static parts. In contrast to that, our intention is to associate with rotors only the fluctuations caused by the dynamical part of the interaction. As shown below, this choice of decoupling provides us with a more convenient description of dynamical screening. The physical effect of the static component of the interaction is of a different nature than the finite-frequency components and it is convenient to treat it separately.

An inverted operator $\bar{U}^{-1}\left(\tau-\tau^{\prime}\right)$ in the expression for $S_{\phi}$ should not be understood in a literal mathematical way. Indeed, such an operator does not exist, because the amplitude of the zeroth mode of $\bar{U}\left(\tau-\tau^{\prime}\right)$, i.e., $\bar{U}(i \nu=0)$, is zero by definition. To impart that operator a definite meaning, we must include into the path integral only such trajectories $\phi(\tau)$ that have no static component: $\int_{0}^{\beta} d \tau \phi(\tau)=0[\phi(i \nu=0)=0]$.

The substitution of spinon-chargon variables into $(12)$ gives an expression for the action which is explicitly split into three parts: an atomic part, a hybridization part, and the part with the dynamical interactions:

$$
\begin{gathered}
S=S_{\mathrm{at}}[\bar{f}, f]+S_{\mathrm{hyb}}[\bar{f}, f ; \theta]+S_{\mathrm{dyn}}[\theta] \\
S_{\mathrm{at}}[\bar{f}, f]=-\sum_{\sigma} \int_{0}^{\beta} d \tau \bar{f}_{\sigma}(\tau)\left[-\partial_{\tau}+\tilde{\mu}\right] f_{\sigma}(\tau)+ \\
+U_{0} \int_{0}^{\beta} d \tau \bar{f}_{\uparrow}(\tau) f_{\uparrow}(\tau) \bar{f}_{\downarrow}(\tau) f_{\downarrow}(\tau) \\
S_{\mathrm{hyb}}[\bar{f}, f ; \theta]= \\
=\sum_{\sigma} \iint_{0}^{\beta} d \tau d \tau^{\prime} \bar{f}_{\sigma}(\tau) \Delta\left(\tau-\tau^{\prime}\right) f_{\sigma}\left(\tau^{\prime}\right) e^{i \theta(\tau)-i \theta\left(\tau^{\prime}\right)} \\
S_{\mathrm{dyn}}[\theta]=\frac{1}{2} \iint_{0}^{\beta} d \tau d \tau^{\prime} \partial_{\tau} \theta(\tau) \bar{U}^{-1}\left(\tau-\tau^{\prime}\right) \partial_{\tau^{\prime}} \theta\left(\tau^{\prime}\right) .
\end{gathered}
$$

This action plays a central role in the formalism being presented. In the limit $\Delta\left(\tau-\tau^{\prime}\right) \rightarrow 0$, fermionic and rotonic degrees of freedom decouple, making the problem exactly solvable. This is the strong-coupling limit of the theory, i.e., the dynamic atomic limit in terms of Ref. 11. The thermal Green's function factorizes into fermionic and rotonic parts if the corresponding degrees of freedom are not coupled in the action:

$$
\begin{array}{r}
G\left(\tau-\tau^{\prime}\right)=G_{f}\left(\tau-\tau^{\prime}\right) G_{X}\left(\tau-\tau^{\prime}\right), \\
G_{f}\left(\tau-\tau^{\prime}\right) \equiv-\left\langle f_{\sigma}(\tau) \bar{f}_{\sigma}\left(\tau^{\prime}\right)\right\rangle \\
G_{X}\left(\tau-\tau^{\prime}\right) \equiv+\left\langle e^{i \theta(\tau)} e^{-i \theta\left(\tau^{\prime}\right)}\right\rangle .
\end{array}
$$

In this specific limit, the low-energy dynamics of fermions is determined by the screened interaction $U_{0}$, and the particular form of $\bar{U}\left(\tau-\tau^{\prime}\right)$ enters only into a bosonic weight-modulating factor in the Green's function. As we will discuss below, the dynamic atomic limit approximation (DALA), as introduced in [11, corresponds to imposing a factorized form with the weight factor given by its atomic limit expression even for finite $\Delta\left(\tau-\tau^{\prime}\right)$. The slave rotor formalism is thus naturally suited for exploring effects beyond the DALA.

\section{APPROXIMATIONS WITHIN THE SLAVE ROTOR PICTURE}

In the atomic limit, $G_{X}$ can be calculated by a direct evaluation of the corresponding path integrals,

$$
G_{X}^{\mathrm{at}}\left(\tau-\tau^{\prime}\right)=\frac{\int \mathcal{D}[\theta] e^{i \theta(\tau)-i \theta\left(\tau^{\prime}\right)-S_{\mathrm{dyn}}[\theta]}}{\int \mathcal{D}[\theta] e^{-S_{\mathrm{dyn}}[\theta]}} .
$$

An expression for a general $2 n$-point correlation function is derived in Appendix A. Here we only give the result 
for the function of two times (see Fig. 1),

$$
G_{X}^{\mathrm{at}}\left(\tau-\tau^{\prime}\right)=\exp \left(-\frac{2}{\beta} \sum_{\nu>0} \frac{\bar{U}(i \nu)}{\nu^{2}}\left[1-\cos \left(\nu\left(\tau-\tau^{\prime}\right)\right)\right]\right) .
$$

The argument of the exponential function in 22 (denoted with $K(\tau)$ in [32]) can be further transformed by substituting Eq. (2) and doing the Matsubara sum,

$$
K(\tau)=\sum_{\alpha} \frac{\lambda_{\alpha}^{2}}{\omega_{\alpha}^{2}} \frac{\cosh \left(\omega_{\alpha}(\tau-\beta / 2)\right)-\cosh \left(\beta \omega_{\alpha} / 2\right)}{\sinh \left(\beta \omega_{\alpha} / 2\right)} .
$$

This nontrivial exponential form of $G_{X}^{\text {at }}(\tau)$ leads to an interesting physical consequence. Let us consider a dynamical interaction function $\bar{U}(i \nu)$ whose spectrum is localized around a single characteristic frequency $\omega_{0}$. The spectrum of the auxiliary function $K(\tau)$ will also have this frequency as a special point. It is then readily seen from a Taylor expansion of the exponent that all multiples of $\omega_{0}$ will be resonances of $G_{X}^{\text {at }}\left(\tau-\tau^{\prime}\right)$; the spectrum of $K(\tau)$ will be replicated along the frequency axis. For a more general case of several characteristic frequencies $\omega_{\alpha}$, the spectrum of $G_{X}^{\text {at }}(\tau)$ will exhibit features at all combinatorial frequencies $\sum_{\alpha} m_{\alpha} \omega_{\alpha}$ with integer coefficients $m_{\alpha}$.

Let us now switch on the hybridization of the impurity electrons with the bath. $f$ fermions and the rotor get coupled through $S_{\text {hyb }}$ and the theory becomes nontrivial. The simplest approximation to treat the model in this case is to artificially suppress the coupling by putting $\theta(\tau)-\theta\left(\tau^{\prime}\right)$ to zero in $S_{\text {hyb }}$ (the phase changes slowly over imaginary time). This approximation was introduced in Ref. 11] as a means to solve dynamic impurity models in the antiadiabatic limit at the cost of static ones and was dubbed the "dynamic atomic limit approximation" (DALA). In DALA, one neglects any mutual influence of $\Delta$ and fluctuations of the full charge, which are induced by the dynamical part of the screened interaction. Obviously, this becomes a good approximation when the energy scales separate, as is the case in the antiadiabatic limit. As follows from Eq. (8), in DALA we have

$$
G(\tau)=G_{f}(\tau ; \Delta) G_{X}^{\text {at }}(-\tau),
$$

where $G_{X}^{\text {at }}$ coincides with 22 and $G_{f}(\tau ; \Delta)$ is calculated for a conventional Anderson model with the given $\Delta$ and the screened static interaction $U_{0}$. The factorized form 24 corresponds to what is called the Bose factor ansatz (BFA) empirically introduced in [11] for the antiadiabatic limit. Interestingly, within the slave rotor formalism, this factorization stands disregarding approximations made on $\theta$ field and without limitations on the parameters of the Hubbard-Holstein model. This comes at the price, however, of introducing a coupling in the differential equations governing the behavior of the two factors, and, in this language, DALA simply corresponds to the zeroth-order approximation in this coupling.

The advantage of DALA is that it provides a simple way to reuse existing quantum impurity solvers designed to work with purely static interactions. Moreover, it substantially simplifies the ill-posed analytic continuation problem. Extracting a spectral function $A(\epsilon)$ from noisy output data of a QMC run for the original AndersonHolstein model is a very difficult task. This is due to the presence of the aforementioned plasmon satellites. On the other hand, the spectral function $A_{f}(\epsilon)$ corresponding to $G_{f}(\tau ; \Delta)$ normally contains only low-energy scales [not larger than $\max \left(\right.$ bandwidth, $\left.U_{0}\right)$ ] and, for this reason, it is much easier to extract using a maximum entropy algorithm. Thanks to Eq. 24, the spectral function of physical electrons is a convolution,

$$
A(\epsilon)=\int_{-\infty}^{+\infty} d \epsilon^{\prime} \frac{\left(1+e^{-\beta \epsilon}\right) A_{f}\left(\epsilon-\epsilon^{\prime}\right) B\left(\epsilon^{\prime}\right)}{\left(1+e^{\beta\left(\epsilon^{\prime}-\epsilon\right)}\right)\left(1-e^{-\beta \epsilon^{\prime}}\right)},
$$

where $B(\epsilon)=-(1 / \pi) \Im G_{X}^{\text {at }}(\epsilon)$ is the spectral function of rotors, which may be calculated from 22 at machine precision.

The main limitation of DALA is that it overlooks the influence of high-energy bosonic fluctuations on lowenergy dynamics of correlated spinons. For example, the coupling of the fermionic degrees of freedom to bosonic fluctuations leads to an "electronic polaron effect," enhancing the mass of the effective low-energy fermionic degrees of freedom or, equivalently, renormalizing the bare hopping matrix elements. This effect has been investigated in detail in Ref. 24 in the framework of LangFirsov transformation techniques. As pointed out in Ref. 11. the simplest way to refine DALA is to take into account an effective an effective change in the magnitude of $\Delta(\tau)$ caused by the bosons. Such a renormalization is described by the Lang-Firsov constant $Z_{B}$ (DALA+LF approximation). As we will see below, the present framework lends itself to an even more refined improvement: the slave rotor formalism can be used to define a dynamical renormalization of the hopping matrix elements, thus generalizing the simple $Z_{B}$ renormalization. Before explaining how to construct such a scheme, we will, however, first need to introduce a generalization of the simple $Z_{B}$ renormalization to finite temperatures. This is done in the following section.

\section{LANG-FIRSOV TRANSFORMATION: EFFECT OF A FINITE TEMPERATURE}

When all bosonic resonances $\omega_{\alpha}$ lie far above energy levels of an isolated atom and the boundary of the conduction band $D$, the interplay of electronic and bosonic fluctuations may be accounted for in a simplified way. This task may be accomplished through construction of a low-energy effective model for electrons.

In this section, we present an effective model derivation based on the Lang-Firsov transformation. This derivation is a finite-temperature generalization of the approach proposed in Ref. [24].

The Lang-Firsov transformation is a unitary change of a basis in the state space of $\hat{H}_{\mathrm{HH}}$. It transforms 

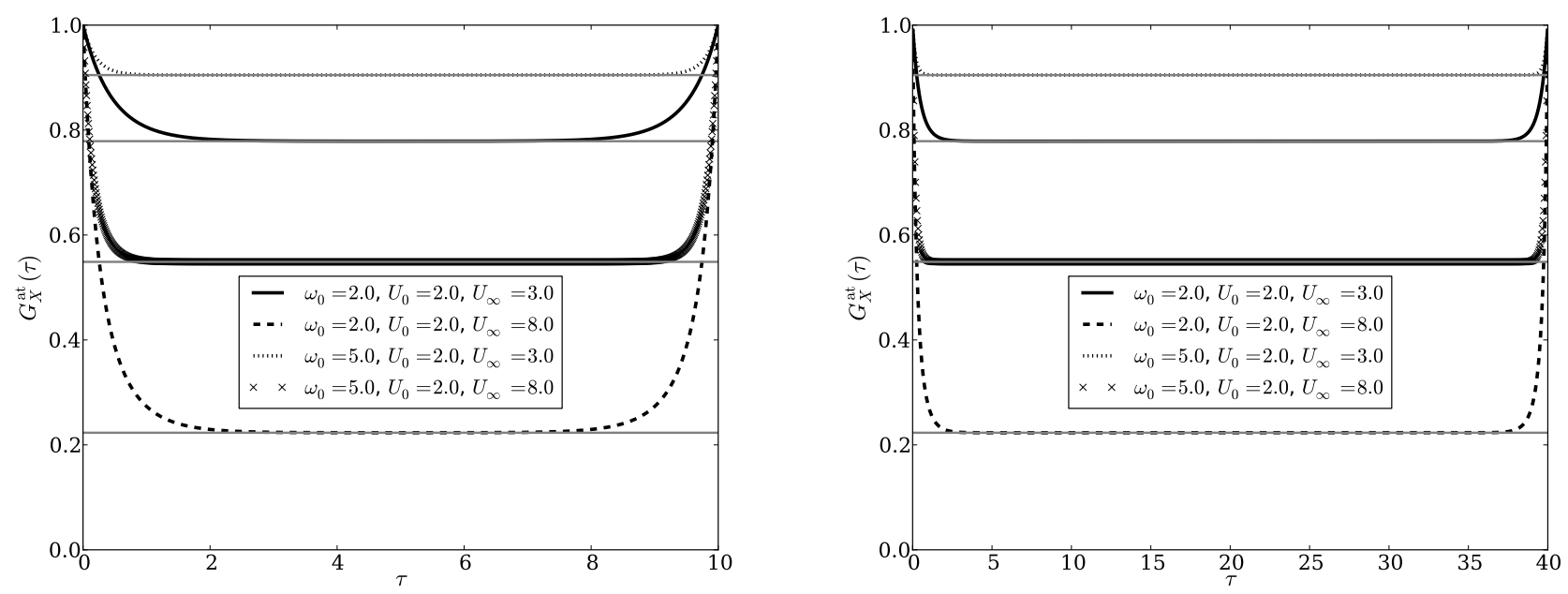

Figure 1: The rotor correlation functions $G_{X}^{\text {at }}(\tau)$ in the atomic limit for the case of a single bosonic mode (left plot: $\beta=10$, right plot: $\beta=40$ ). Horizontal lines show the corresponding values of $Z_{B}$.

the Hamiltonian and field operators, replacing electrons $d_{i \sigma}^{\dagger}, d_{i \sigma}$ with polarons $c_{i \sigma}^{\dagger}, c_{i \sigma}$. The unitary transformation operator is

$$
\hat{U}_{\mathrm{LF}}=\exp \left(-\frac{\lambda}{\omega_{0}} \sum_{i, \alpha, \sigma} d_{i \sigma}^{\dagger} d_{i \sigma}\left(b_{i \alpha}-b_{i \alpha}^{\dagger}\right)\right) .
$$

The field operators transform as follows:

$$
\begin{aligned}
& c_{i \sigma}=\hat{U}_{\mathrm{LF}} d_{i \sigma} \hat{U}_{\mathrm{LF}}^{\dagger}=d_{i \sigma} \exp \left(\sum_{\alpha} \frac{\lambda_{\alpha}}{\omega_{\alpha}}\left(b_{i \alpha}-b_{i \alpha}^{\dagger}\right)\right) \\
& c_{i \sigma}^{\dagger}=\hat{U}_{\mathrm{LF}} d_{i \sigma}^{\dagger} \hat{U}_{\mathrm{LF}}^{\dagger}=d_{i \sigma}^{\dagger} \exp \left(-\sum_{\alpha} \frac{\lambda_{\alpha}}{\omega_{\alpha}}\left(b_{i \alpha}-b_{i \alpha}^{\dagger}\right)\right) .
\end{aligned}
$$

The Hamiltonian is completely equivalent to $\hat{H}_{\mathrm{HH}}$, although it does not explicitly contain a coupling term between polarons and bosons 3435 .

$$
\begin{aligned}
\hat{H}_{\mathrm{LF}}=\hat{U}_{\mathrm{LF}} \hat{H}_{\mathrm{HH}} \hat{U}_{\mathrm{LF}}^{\dagger}= & \\
= & -\sum_{i j, \sigma} t_{i j} c_{i \sigma}^{\dagger} c_{j \sigma}-\tilde{\mu} \sum_{i \sigma} c_{i \sigma}^{\dagger} c_{i \sigma}+ \\
& +U_{0} \sum_{i} c_{i \uparrow}^{\dagger} c_{i \uparrow} c_{i \downarrow}^{\dagger} c_{i \downarrow}+\sum_{i, \alpha} \omega_{\alpha} b_{i \alpha}^{\dagger} b_{i \alpha} .
\end{aligned}
$$

The Lang-Firsov transformation results in a renormalization of the chemical potential $\tilde{\mu}$ and the unscreened part of the interaction $U_{0}$; these quantities coincide with those in 10.

The polaron degrees of freedom represent electrons dressed by bosonic fluctuations. One can take this dressing into account in an approximate way by calculating renormalized hopping constants $Z_{B} t_{i j}$ for the original electrons. In the paper by Casula et al.$^{24}$ this is done by projecting the Lang-Firsov Hamiltonian onto the subspace of zero-boson states,

$$
\hat{H}_{\text {eff }}=\left\langle\{0\}_{\alpha}\left|\hat{H}_{\mathrm{LF}}\right|\{0\}_{\alpha}\right\rangle \text {, given } \omega_{\alpha} \gg U_{0}, t_{i j} .
$$

This leads to the following effective low-energy Hamiltonian:

$$
\begin{aligned}
\hat{H}_{\mathrm{eff}}=-\sum_{i j, \sigma} Z_{B} t_{i j} d_{i \sigma}^{\dagger} d_{j \sigma} & -\tilde{\mu} \sum_{i \sigma} d_{i \sigma}^{\dagger} d_{i \sigma}+ \\
& +U_{0} \sum_{i} d_{i \uparrow}^{\dagger} d_{i \uparrow} d_{i \downarrow}^{\dagger} d_{i \downarrow}
\end{aligned}
$$

which is a conventional Hubbard model with a renormalized bandwidth, interaction strength, and chemical potential, but where the one-particle hopping matrix elements have been renormalized by a factor $Z_{B}<1$ that we discuss below.

This simple renormalization is valid in the antiadiabatic regime and for not very high temperatures. As the temperature goes higher, more boson excitations are effectively created and the projection onto the zero-boson subspace becomes no longer valid.

When the effective low-energy model is used to calculate a spectrum of physical electrons, the Green's function reads

$$
G_{i \sigma ; j \sigma}^{\text {low-energy }}(\tau)=-Z_{B}\left\langle\mathbb{T} d_{i \sigma}(\tau) d_{j \sigma}^{\dagger}(0)\right\rangle_{\hat{H}_{\text {eff }}} .
$$

The full spectral weight, corresponding to a Green's function defined in this way, is equal to $Z_{B}$. The remaining contribution $1-Z_{B}$ to the weight is constituted by high-frequency scattering processes involving boson creations/annihilations. As we have excluded such processes from $\hat{H}_{\text {eff }}$, consistency of the theory requires us to diminish the spectral weight accordingly. 
Here we present a finite-temperature expression for $Z_{B}$, which is obtained by tracing out all bosonic degrees of freedom from $\hat{H}_{\mathrm{LF}}$ (a complete derivation is found in Appendix B,

$$
\begin{aligned}
Z_{B}= & \exp \left(-\sum_{\alpha} \frac{\lambda_{\alpha}^{2}}{\omega_{\alpha}^{2}} \operatorname{coth}\left(\beta \omega_{\alpha} / 2\right)\right)= \\
& =\exp \left(\frac{1}{\pi} \int_{0}^{+\infty} \frac{\Im U_{\text {ret }}(\epsilon)}{\epsilon^{2}} \operatorname{coth}(\beta \epsilon / 2) d \epsilon\right) .
\end{aligned}
$$

Expression (33) contains two dimensionless combinations of three energy scales, namely, $\lambda_{\alpha} / \omega_{\alpha}$ and $\beta \omega_{\alpha}$. They give rise to a multitude of temperature limiting cases, some of which we analyze here.

- $\beta \rightarrow \infty ; \lambda, \omega=$ const. This is the zero-temperature limit, consistent with the result of Casula et al., $Z_{B}=\exp \left(-\sum_{\alpha} \lambda_{\alpha}^{2} / \omega_{\alpha}^{2}\right)$.

- $\beta \rightarrow 0 ; \beta \omega=$ const. If all energy scales of the bosonic subsystem follow a rise of the temperature, then our expression stands for arbitrarily small $\beta$. However, this case is usually of little physical interest.

- $\beta \rightarrow 0 ; \lambda, \omega=$ const. In this more physically relevant case, we get the curious result that $Z_{B}$ must vanish together with beta. The system rapidly falls down to the atomic limit as the temperature grows. It seems that $Z_{B}$ alone can provide a reasonable effective description only for not very small values of $\beta \omega_{\alpha}$. One can use the condition $Z_{B} \lesssim 1$ to determine the order of magnitude of the temperature where a description in terms of $Z_{B}$ is still valid. Beyond this region, a more refined theory of dynamical screening is needed.

Of course, it is far from obvious that the calculated temperature dependence of $Z_{B}$ plays any role in practical applications. Indeed, for realistic plasmon frequencies $\omega_{\alpha} \simeq 10 \mathrm{eV}$, the temperature which could grant considerable boson excitation probabilities would be too high to observe interesting electron correlation effects. Nonetheless, the obtained expression will turn out to be useful for finding a connection between the Lang-Firsov trick and the formalism presented below.

How can these insights be used within the slave rotor framework employed as a solver technique for the DMFT equations with dynamical interactions? A renormalization of the hopping matrix translates into a renormalization of the hybridization function $\Delta(i \omega)$, when a Hubbard-Holstein model (or an equivalent Hubbard model with a dynamical interaction) is mapped onto the Anderson model by DMFT. The effective renormalization of $\Delta\left(\tau-\tau^{\prime}\right)$ in the slave rotors picture is caused by a coupling term, $\Delta\left(\tau-\tau^{\prime}\right) e^{i \theta(\tau)-i \theta\left(\tau^{\prime}\right)}$. Obviously, the $Z_{B}$ factor thus corresponds to a specific approximation to the second factor in this expression. One could, for example, think of the following mean-field estimate of the renormalization constant $Z_{B}$ :

$$
Z_{B}=\frac{1}{\beta} \int_{0}^{\beta} d \tau\left\langle e^{-i \theta(\tau)+i \theta(0)}\right\rangle_{\mathrm{at}}
$$

where the subscript "at" indicates that the average value is taken with the atomic limit action.

The imaginary-time integral in this expression means that we are interested only in the low-energy fluctuations of the $\theta$ field. Substituting $G_{X}^{\text {at }}(\tau)$ into this expression, we rewrite the integral,

$$
\begin{aligned}
Z_{B}=\exp & \left(-\sum_{\alpha} \frac{\lambda_{\alpha}^{2}}{\omega_{\alpha}^{2}} \operatorname{coth}\left(\beta \omega_{\alpha} / 2\right)\right) \times \\
& \times \int_{-1 / 2}^{+1 / 2} d x \exp \left[\sum_{\alpha} \frac{\lambda_{\alpha}^{2}}{\omega_{\alpha}^{2}} \frac{\cosh \left(\beta \omega_{\alpha} x\right)}{\sinh \left(\beta \omega_{\alpha} / 2\right)}\right]
\end{aligned}
$$

If the adiabatic ratios $\lambda_{\alpha} / \omega_{\alpha}$ are all small, the right-hand side integral goes to 1 , and our estimate of $Z_{B}$ becomes consistent with the antiadiabatic-limit expression (33). While we could use the improved estimate 35 for $Z_{B}$ to try reaching the $\lambda_{\alpha} / \omega_{\alpha} \simeq 1$ region in practical calculations, we prefer to go a bit further. In the next section, we introduce an approximation beyond DALA, in which $\Delta$ undergoes a dynamic rather then a static renormalization.

\section{BEYOND DALA: THE OPTIMAL MEAN-FIELD TREATMENT OF $S_{\text {hyb }}$}

We now come to the central idea of the present work. The above discussion has addressed how dynamical screening leads to a mass enhancement of the lowenergy fermionic degrees of freedom, as expressed by the bosonic renormalization factor $Z_{B}$. It also became clear, however, that replacing the truly dynamical couplings between fermionic and rotor degrees of freedom by a simple number introduces simplifications that are justified only in specific limits. In this section, we will derive a more general dynamical renormalization scheme, based on the slave rotor framework. More precisely, we will construct an (in a sense to be specified) optimal bosonic renormalization factor.

To this end, we consider a class of effective spinon-only models with modified hybridization functions $\tilde{\Delta}(\tau)$,

$$
\tilde{S}_{\mathrm{hyb}}[\bar{f}, f]=\iint_{0}^{\beta} d \tau d \tau^{\prime} \sum_{\sigma} \bar{f}_{\sigma}(\tau) \tilde{\Delta}\left(\tau-\tau^{\prime}\right) f_{\sigma}\left(\tau^{\prime}\right)
$$

Whenever an action $S$ of a physical system is replaced by a simpler "trial" action $\tilde{S}$, it is convenient to apply Feynman's variational criterion to estimate what is the best choice of the parameters of $\tilde{S}$,

$$
\mathcal{F}(\tilde{\Delta})=\langle S-\tilde{S}\rangle_{\tilde{S}}-\ln \tilde{Z}+\ln Z=\min ,
$$




$$
\tilde{Z} \equiv \int e^{-\tilde{S}} \mathcal{D}[\bar{f}, f], \quad Z \equiv \int e^{-S} \mathcal{D}[\bar{f}, f]
$$

We are going to calculate the variation of Feynman's functional with respect to $\tilde{\Delta}(\tau)$ which parametrizes the trial action $\tilde{S}=S_{\text {at }}+\tilde{S}_{\text {hyb }}+S_{\text {dyn }}$. Doing so, we obtain an extremum condition,

$$
\begin{aligned}
& \iint_{0}^{\beta} d \tau^{\prime \prime} d \tau^{\prime \prime \prime} \sum_{\sigma} \frac{\delta \tilde{G}_{f, \sigma \sigma}\left(\tau^{\prime \prime \prime}-\tau^{\prime \prime}\right)}{\delta \tilde{\Delta}\left(\tau-\tau^{\prime}\right)} \times \\
& \times\left[\tilde{\Delta}\left(\tau^{\prime \prime \prime}-\tau^{\prime \prime}\right)-\Delta\left(\tau^{\prime \prime \prime}-\tau^{\prime \prime}\right) G_{X}^{\mathrm{at}}\left(\tau^{\prime \prime \prime}-\tau^{\prime \prime}\right)\right]=0 .
\end{aligned}
$$

An apparent solution of this equation is $\tilde{\Delta}(\tau)=$ $\Delta(\tau) G_{X}^{\text {at }}(\tau)$. This means that the atomic limit estimate $\left\langle e^{i \theta(\tau)-i \theta\left(\tau^{\prime}\right)}\right\rangle \simeq G_{X}^{\text {at }}\left(\tau-\tau^{\prime}\right)$ is indeed the best "modulation" function within the proposed ansatz. The procedural change from DALA+LF to the proposed approximation consists of using $G_{X}^{\text {at }}\left(\tau-\tau^{\prime}\right)$ instead of $Z_{B}$ as a prefactor of the hybridization function. Such a change does not introduce much additional complication, yet it allows one to achieve better results for intermediate values of the adiabatic parameter.

The proposed approach is summarized in Fig. 2, The part of the scheme inside the dashed box is a DMFT loop involving only spinon degrees of freedom. The effective Anderson impurity model is solved with an impurity model solver for static interactions, e.g., a standard continuous-time quantum Monte Carlo (CTQMC) solver. The screened values $U_{0}, \tilde{\mu}$ and an arbitrary initial guess for $\Delta$ are used as input parameters of the first solver run. The resultant spinon Green's function $G_{f}(\tau)$ is then multiplied by $G_{X}^{\text {at }}(\tau)$ in order to obtain the impurity Green's function of physical electrons.

In the next step, a standard DMFT self-consistency procedure is performed and an updated hybridization function $\Delta(\tau)$ for the electrons is constructed. Within the optimized BFA approximation, $\Delta(\tau)$ is replaced by $\tilde{\Delta}(\tau)=\Delta(\tau) G_{X}^{\text {at }}(\tau)$. In this modulated form, the hybridization function is used as input data for the next solver run. The loop is repeated until $G_{f}(\tau)$ converges with a prescribed accuracy.

Schemes for DALA and DALA+LF would differ only in the way $\tilde{\Delta}(\tau)$ is obtained from $\Delta(\tau)$ (multiplication by 1 and $Z_{B}$, respectively).

\section{INTERPRETATION OF THE "OPTIMAL BOSE FACTOR APPROACH"}

Before heading to the actual results, we would like to discuss the physical meaning of the modifications included in the "optimal Bose factor approach." To this end, we start from the DMFT self-consistency condition, which - for a lattice with a bare dispersion law of elec-

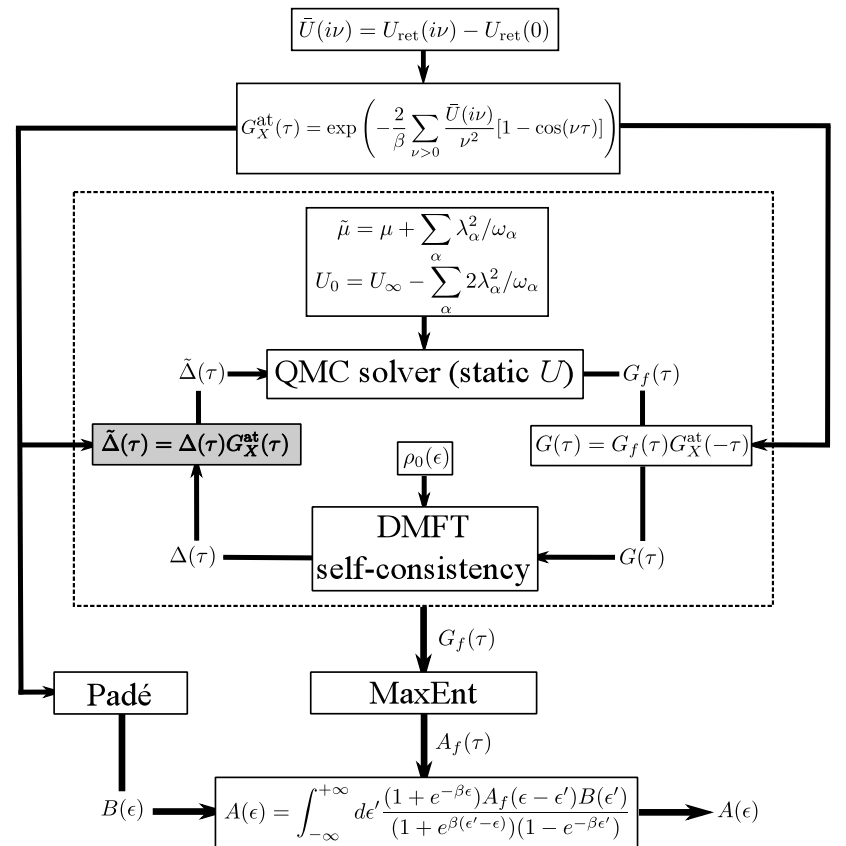

Figure 2: Scheme of the DMFT loop within the approximation proposed in Sec. VI. The highlighted box would be $\tilde{\Delta}(\tau)=\Delta(\tau)$ in DALA and $\tilde{\Delta}(\tau)=Z_{B} \Delta(\tau)$ in DALA+LF.

trons $\epsilon=\varepsilon(\vec{k})$-reads:

$$
\begin{aligned}
& \frac{1}{i \omega_{n}+\mu-\Delta\left(i \omega_{n}\right)-\Sigma_{\mathrm{AHM}}\left(i \omega_{n}\right)}= \\
& =\frac{1}{\Omega_{\mathrm{BZ}}} \sum_{\vec{k}} \frac{1}{i \omega_{n}+\mu-\varepsilon(\vec{k})-\Sigma_{\mathrm{AHM}}\left(i \omega_{n}\right)} .
\end{aligned}
$$

Here, $\Sigma_{\mathrm{AHM}}\left(i \omega_{n}\right)$ is the self-energy of the auxiliary Anderson-Holstein model. Unfortunately, there is no simple form of this expression written in terms of $\tilde{\Delta}$, $G_{f}$, and $G_{X}^{\text {at }}$; any such equation in the frequency domain would inevitably contain convolutions, but not products. However, for the particular case of the Bethe lattice with infinite coordination number the actual equation simplifies to

$$
\tilde{\Delta}(\tau)=t^{2}\left(G_{X}^{\mathrm{at}}(\tau)\right)^{2} G_{f}(\tau)
$$

i.e., the DMFT-loop for the spinons is built using a chargon-screened hopping parameter $t(\tau) \equiv t G_{X}^{\text {at }}(\tau)$. For comparison, in DALA one has the screening coefficient equal to $\sqrt{G_{X}^{\text {at }}(\tau)}$ and, in DALA $+\mathrm{LF}$, to $\sqrt{Z_{B} G_{X}^{\text {at }}(\tau)}$.

The proposed optimized Bose factor ansatz has an important advantage over DALA+LF: It leads to a redistribution of the spectral weight of $\Delta(\tau)$ rather than to a change of the full weight. Indeed, the full weight is conserved and is equal to a discontinuous jump of $\tilde{\Delta}(\tau)$ at zero time:

$$
\begin{array}{r}
\tilde{\Delta}(-0)-\tilde{\Delta}(+0)=\Delta(-0) G_{X}^{\mathrm{at}}(-0)-\Delta(+0) G_{X}^{\mathrm{at}}(+0)= \\
=\Delta(-0)-\Delta(+0) .
\end{array}
$$



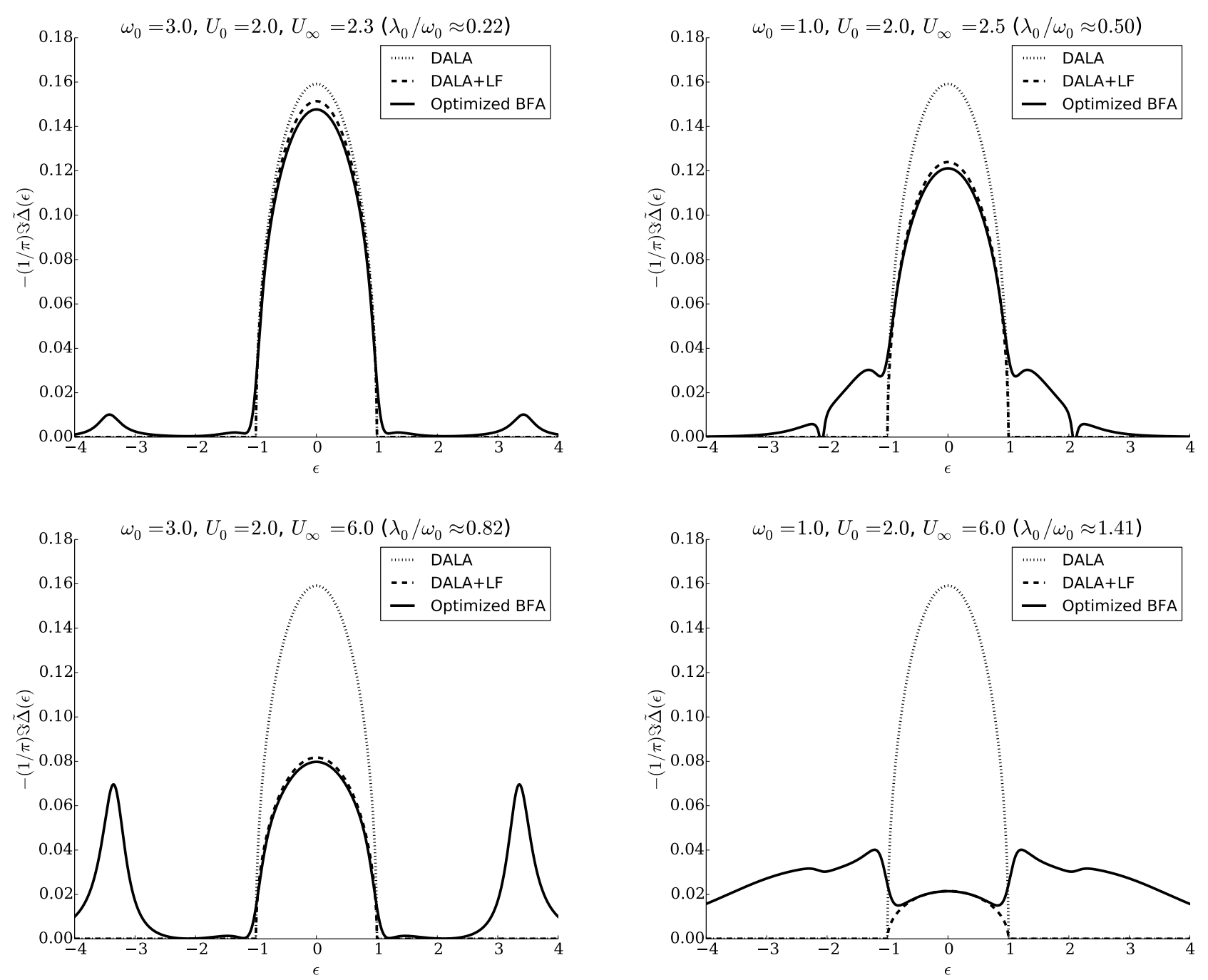

Figure 3: Spectral functions corresponding to $\tilde{\Delta}(\tau)$ at the first iteration of the self-consistency loop within different approximations and for different values of the adiabatic parameter $\lambda_{0} / \omega_{0}(\beta=20)$ ). The unmodulated (or, equivalently, DALA) spectral function is defined to have a semielliptic shape, $\rho(\epsilon)=\theta(1-|\epsilon|) \sqrt{1-\epsilon^{2}} / 2 \pi$.

One can thus think of the approximation as replacing the simplistic band renormalization by a weightconserving transformation of the noninteracting density of states. We illustrate this fact by plotting in Fig. 3 the modifications induced onto the hybridization function corresponding to a model with semielliptic density of states. This quantity thus corresponds to the effective hybridization function for the Bethe lattice $-\tilde{\Delta}(\epsilon)$ as defined in Eq. (40) — at the first iteration of the abovediscussed self-consistency loop. As seen in the Figure, there is always a low-frequency region of the density of states (DOS) (perhaps narrow enough) in which the effect of DALA+LF (multiplication by $Z_{B}$ ) is almost the same as from the optimized BFA. However, the disagreement grows stronger outside this region, as a value of the adiabatic parameter $\lambda_{0} / \omega_{0}$ increases. In the deep antiadiabatic limit (upper left plot), only a small part of the spectral weight is transferred to the plasmonic satellites and DALA+LF indeed works well. For a larger value of $\lambda_{0} / \omega_{0}$ and a small characteristic frequency (upper right plot), a substantial part of the spectral weight is pulled out from the center of the conduction band. It is then transferred to a newly formed pair of "wings," which effectively extend the bandwidth. The most drastic change of the DOS occurs in the intermediate and adiabatic regimes (lower plots). Not only is a major part of the spectral weight transferred to the plasmonic satellites, but also the conduction band may completely change its shape and widen due to merging with the satellites. 


\section{TECHNICALITIES}

To solve the effective impurity model, we employed a hybridization expansion Monte Carlo solver (CT-HYB), which is part of the TRIQS application suite ${ }^{42}$. The result of the impurity solver is a Green's function in imaginary time, which is dressed by $G_{X}(-\tau)$ according to the BFA (see Fig. 2).

A note should be made about the analytic continuation procedure used. We use Padé approximants to reconstruct the bosonic spectral function $B(\epsilon)$ and Sandvik's stochastic algorithm ${ }^{43}$ for the spectrum of spinons $A_{f}(\epsilon)$. Doing the convolution $(25)$ proves to be problematic due to the divergence of the Bose-Einstein distribution at zero energy. To circumvent this difficulty, we use an auxiliary spectral function $\tilde{B}(\epsilon)$, reconstructed from $G_{X}^{\text {at }}(\tau)-Z_{B}$. By construction, $\tilde{B}(\epsilon)$ has a zero at $\epsilon=0$, which compensates the divergence of the integral kernel. The resulting density of states is immediately obtained as $A(\epsilon)=A_{f} * \tilde{B}+Z_{B} A_{f}(\epsilon)$ (convolution in the sense of 25].

\section{COMPARISON OF DIFFERENT APPROACHES}

In order to compare the three schemes described in the previous sections (DALA, DALA+LF, and the optimized BFA), we have performed several series of DMFT runs for the Hubbard-Holstein model on a Bethe lattice.

The unit of energy is set to the half bandwidth of the bare dispersion of the lattice. Each of the six cases is defined by an unscreened value of on-site interaction $U_{\infty}$ and by parameters of a single bosonic resonance $\left(\lambda_{0}, \omega_{0}\right)$. Inclusion of more bosonic modes would not require a significantly larger numerical effort. However, the resulting spectra are easier to interpret if only one bosonic excitation is present. The adiabatic parameter $\lambda_{0} / \omega_{0}$ is varying from 0.1 (truly antiadiabatic regime) to 0.8 (intermediate regime).

In all cases, the value of $U_{\infty}$ is chosen in a way to put the DMFT loop close to the paramagnetic Mott transition point: $U_{0}=2.4, \beta=60$ [44. We perform calculations at half filling, by choosing the chemical potential as $\mu=U_{\infty} / 2-2 \lambda_{0}^{2} / \omega_{0}$, such as to ensure the particle-hole symmetry of the effective Anderson model.

The spectral functions are shown in Fig. 4. The insets depict imaginary-time Green's functions $G_{f}(\tau)$, which are directly measured by the CT-HYB solver. Figures 4(a)-4(c) show the cases where DALA, DALA+LF, and the optimized BFA agree on the metallic type of the solution. For a very high plasmon frequency and a small value of the adiabatic parameter [Fig. 4(a)], the satellites are barely visible; as the frequency approaches $U_{0} / 2$, the satellites become more pronounced. Spectra depicted in Figs. 4(d)-4(f) are of the insulator type as seen by DALA+LF and by the optimized BFA, but not by DALA. Depending on the plasmon frequency, the satellites are either completely masked by the Hubbard subbands [Fig. 4(d)] or well pronounced and contain a considerable part of the spectral weight [Fig. 4(e)]. In the extreme case of Fig. 4(f) of a low frequency but strongly coupled bosons, the spectrum is comblike with the shape of the "teeth" replicating the Hubbard bands.

As one can see, there is a qualitative difference between DALA and the other two approximations, which is easily understood. DALA tends to underestimate the reduction of the DOS at the Fermi level caused by the bosons, and is thus biased towards the metallic phase. At the same time, differences between DALA+LF and the optimized BFA are quite subtle, if visible. The discrepancy could be more pronounced at higher temperatures, where $G_{X}^{\text {at }}(\tau)$ would be approximately constant on a smaller part of the interval $[0 ; \beta]$ (see Fig. 11). This issue is illustrated by spectral functions at higher temperatures presented in Figs. $5(\beta=10)$ and $6(\beta=3)$. The differences between DALA+LF and the optimized BFA seem to be of the highest importance, when energies of the bosonic resonance $\omega_{0}$ and of the atomic level $U_{0} / 2$ are comparable.

Finally, we discuss an interesting feature of the physical spectral functions $A(\epsilon)$ plotted in Fig. 4. Indeed, in a Fermi liquid with local self-energy - in its coherent low-temperature regime - one expects the value of the spectral function on the Fermi level to coincide with the value of the noninteracting density of states. This "pinning condition" is violated in our spectra. We checked that this is a finite-temperature effect which is more pronounced in the optimized BFA calculations as compared to the DALA results. The "better" pinning is, however, an artifact of the underestimated correlation strength by DALA.

\section{SUMMARY AND PERSPECTIVES}

In the present paper, we have introduced a systematic approach to the Hubbard-Holstein model, inspired by the slave rotor transformation proposed by Florens and Georges.

We have given a derivation and clarified the physical meaning of existing methods, such as the dynamic atomic limit approximation (DALA) and DALA combined with a Lang-Firsov procedure (DALA+LF). DALA, being an effective tool to describe dynamic screening in solids, was originally derived from an intuitively chosen ansatz. DALA+LF is an improved version of DALA, which better describes the boson-induced narrowing of the conduction band. However, DALA+LF suffers from a spectral weight loss issue which should be treated with care. The proposed approach demonstrates that both DALA and DALA+LF can be understood as simple approximations made on the fluctuating rotor-dependent factor $\exp \left(i \theta(\tau)-i \theta\left(\tau^{\prime}\right)\right)$

Apart from this, we have found an approximation to the exponential factor, which is optimal in the sense of Feynman's variational criterion. This "optimized BFA" 


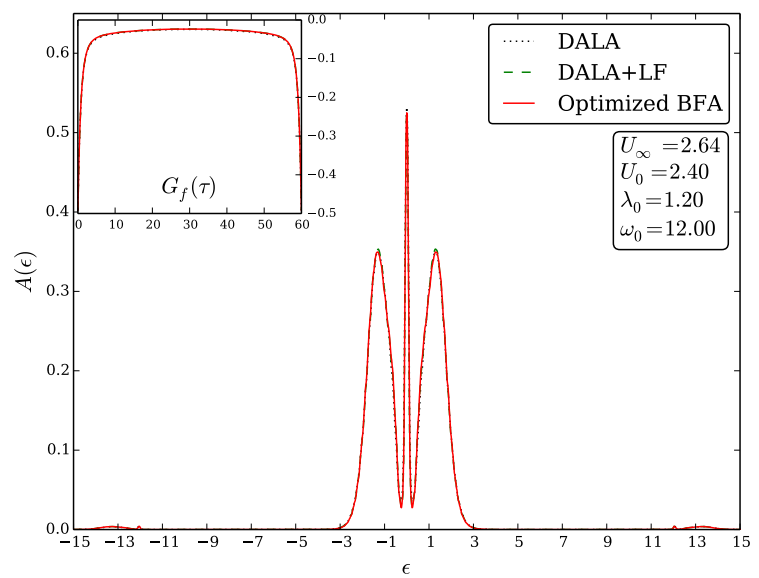

(a) $\lambda_{0} / \omega_{0}=0.1$

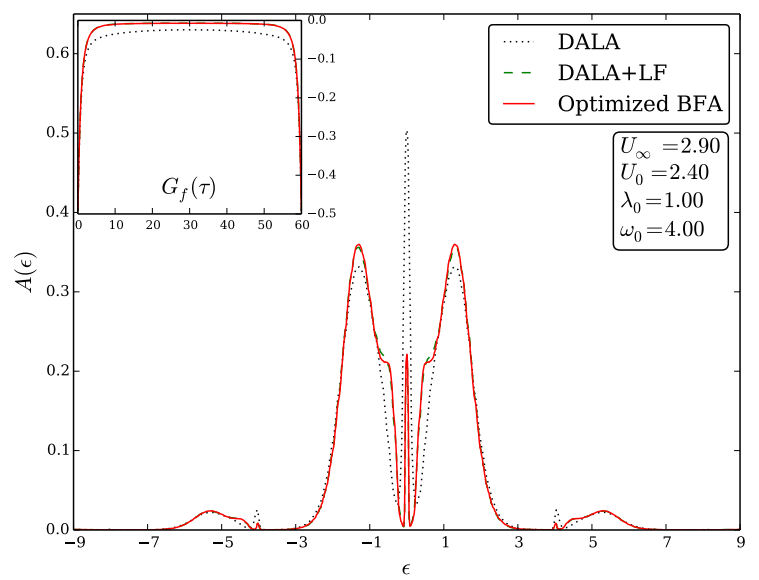

(c) $\lambda_{0} / \omega_{0}=0.25$

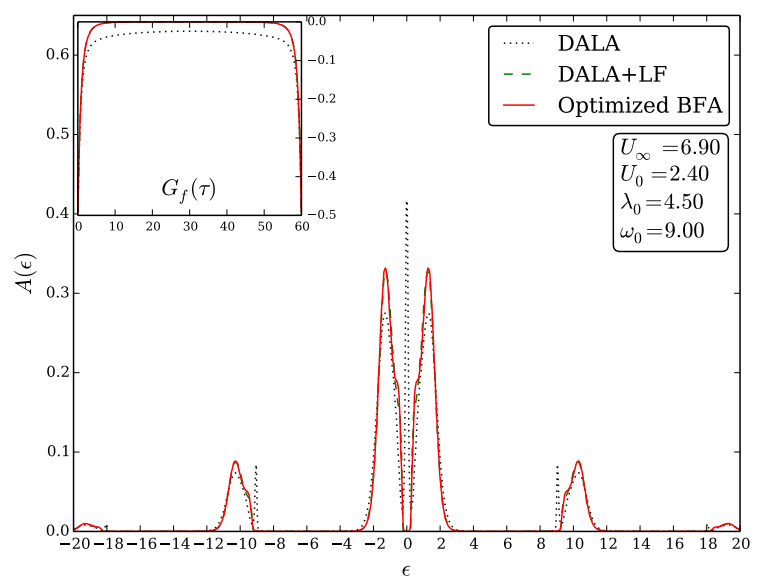

(e) $\lambda_{0} / \omega_{0}=0.5$

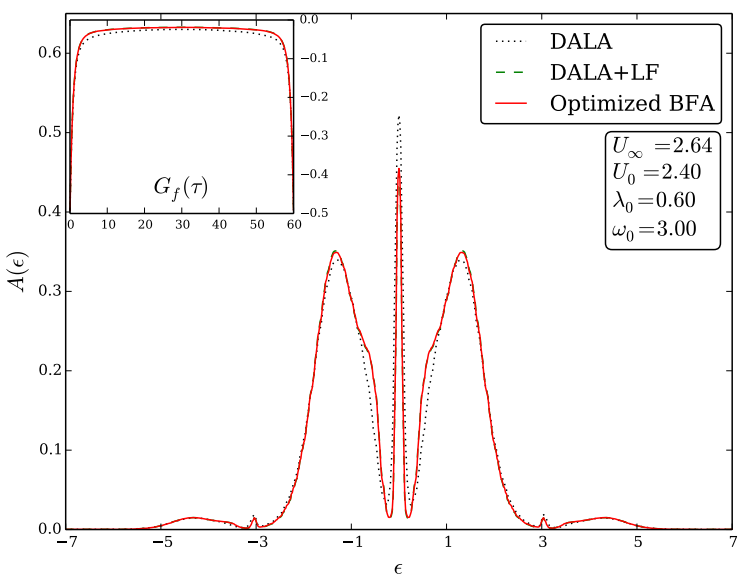

(b) $\lambda_{0} / \omega_{0}=0.2$

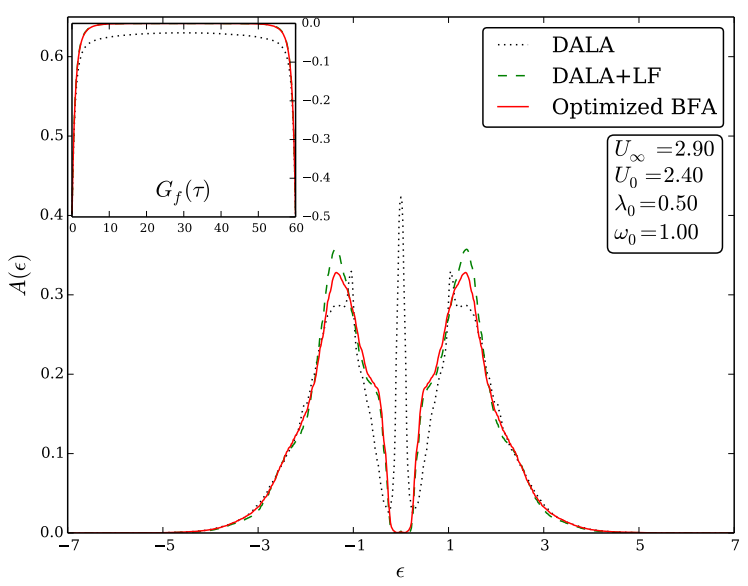

(d) $\lambda_{0} / \omega_{0}=0.5$

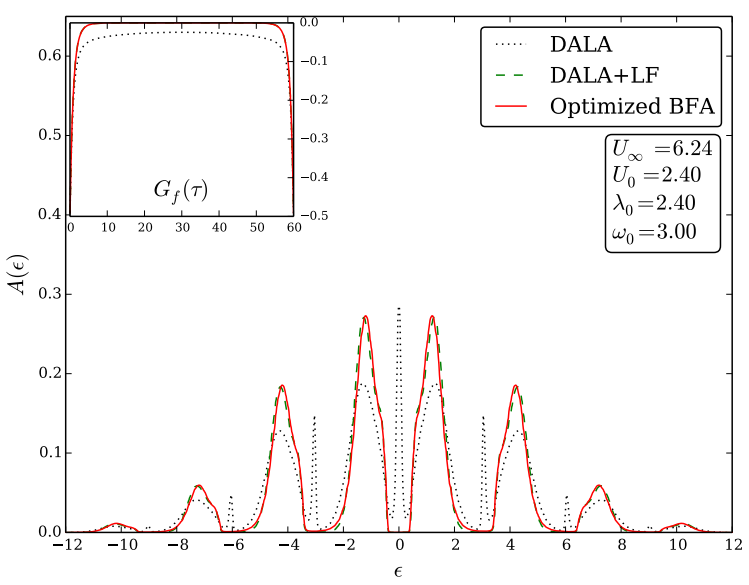

(f) $\lambda_{0} / \omega_{0}=0.8$

Figure 4: Local spectral functions of the Hubbard-Holstein model calculated within three different approximations and for six sets of parameters. Spinon Green's functions $G_{f}(\tau)$ produced by the DMFT loop are shown in an inset. 


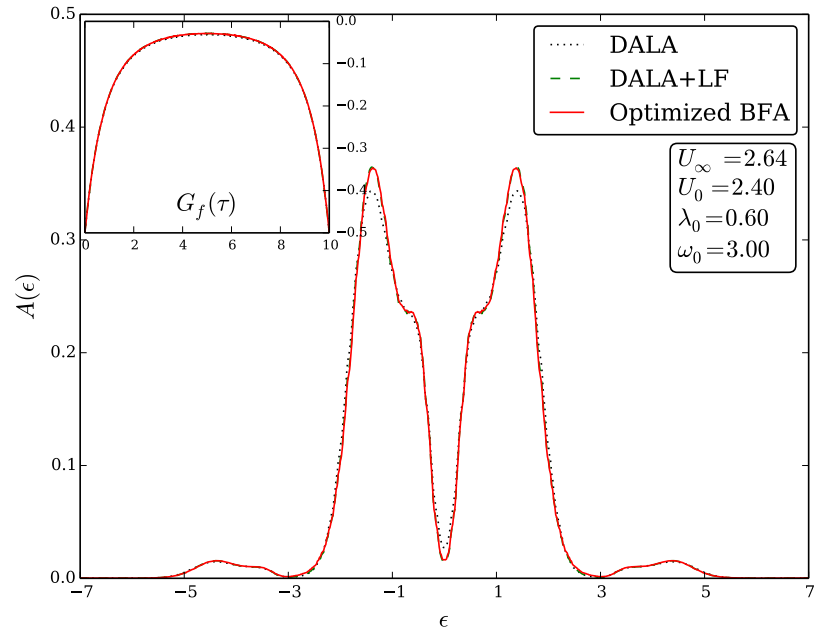

(a) $\lambda_{0} / \omega_{0}=0.2$

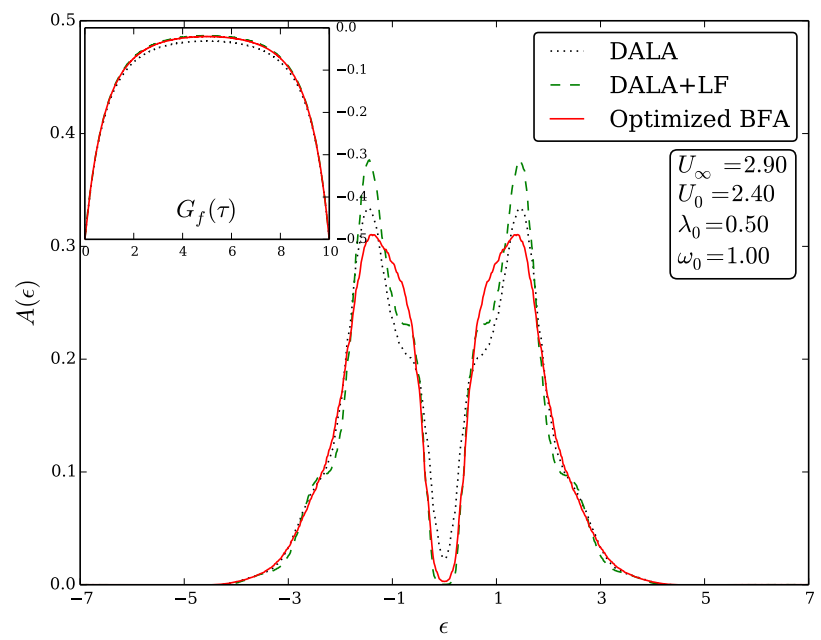

(b) $\lambda_{0} / \omega_{0}=0.5$

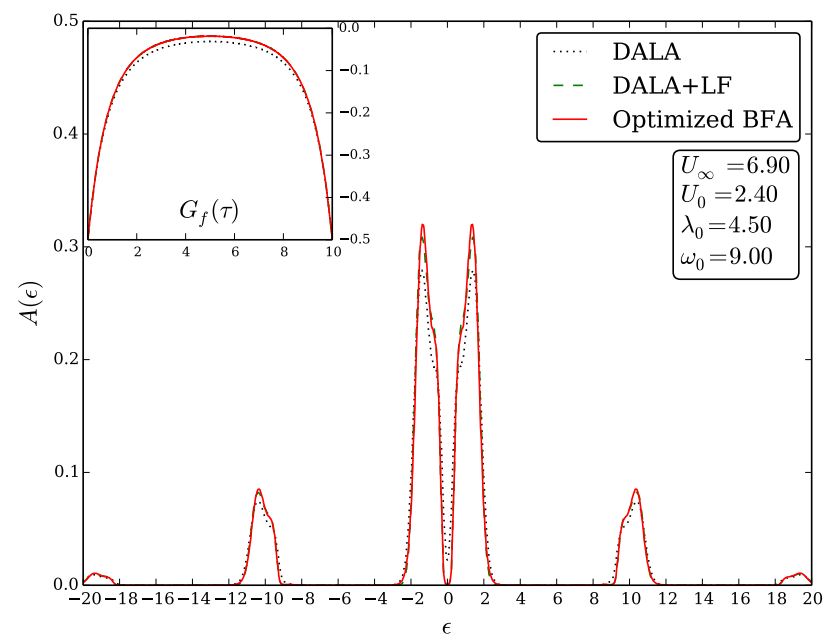

(c) $\lambda_{0} / \omega_{0}=0.5$

Figure 5: Local spectral functions of the Hubbard-Holstein model $(\beta=10)$. Insets: Spinon Green's functions $G_{f}(\tau)$ produced by the DMFT loop.

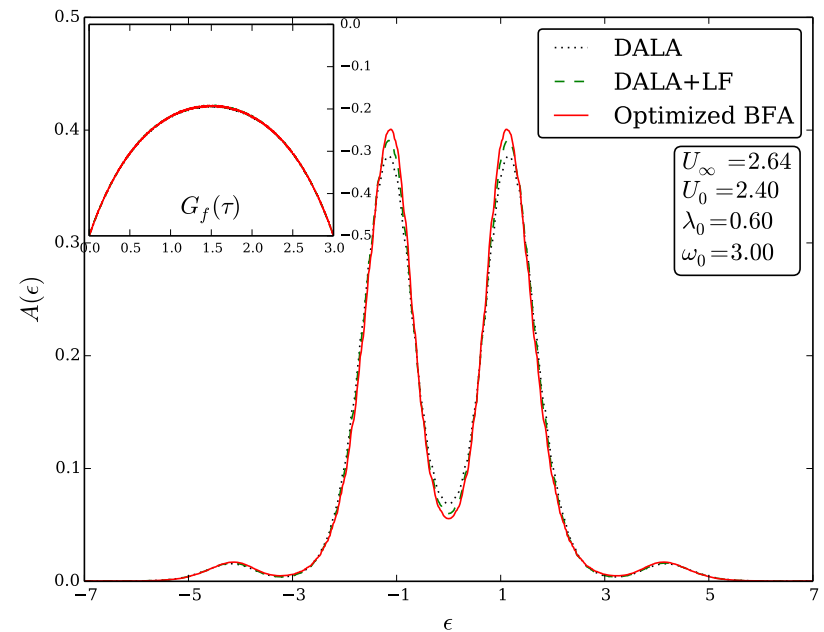

(a) $\lambda_{0} / \omega_{0}=0.2$

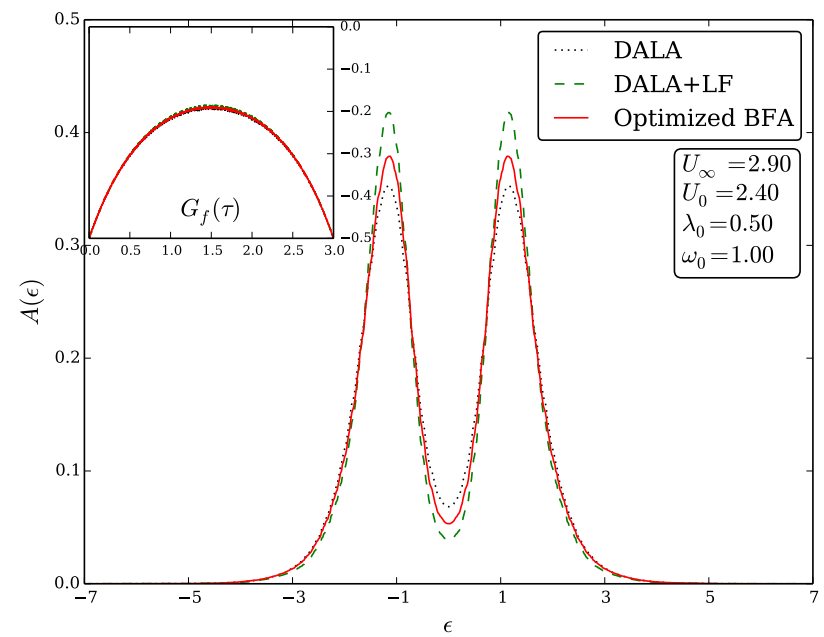

(b) $\lambda_{0} / \omega_{0}=0.5$

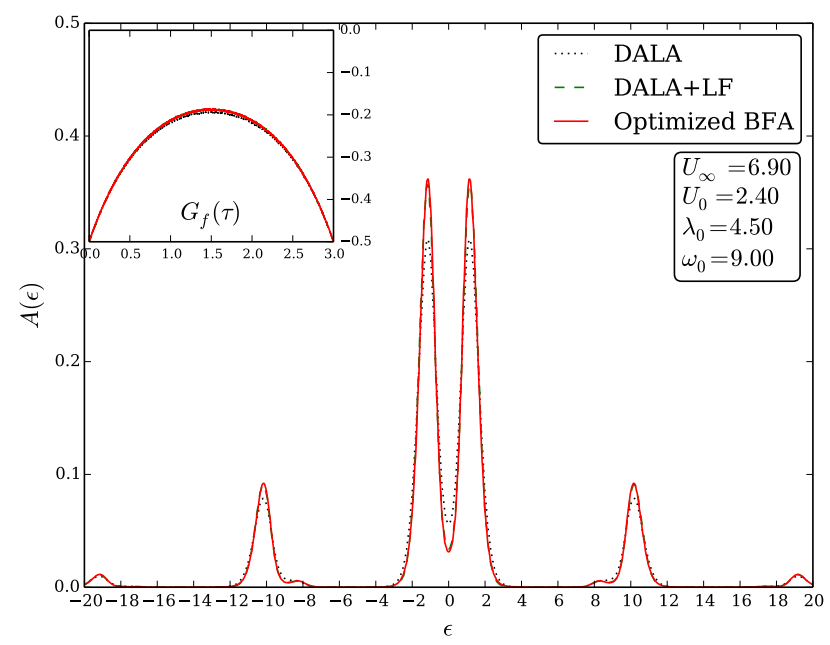

(c) $\lambda_{0} / \omega_{0}=0.5$

Figure 6: Local spectral functions of the Hubbard-Holstein model $(\beta=3)$. Insets: Spinon Green's functions $G_{f}(\tau)$ produced by the DMFT loop. 


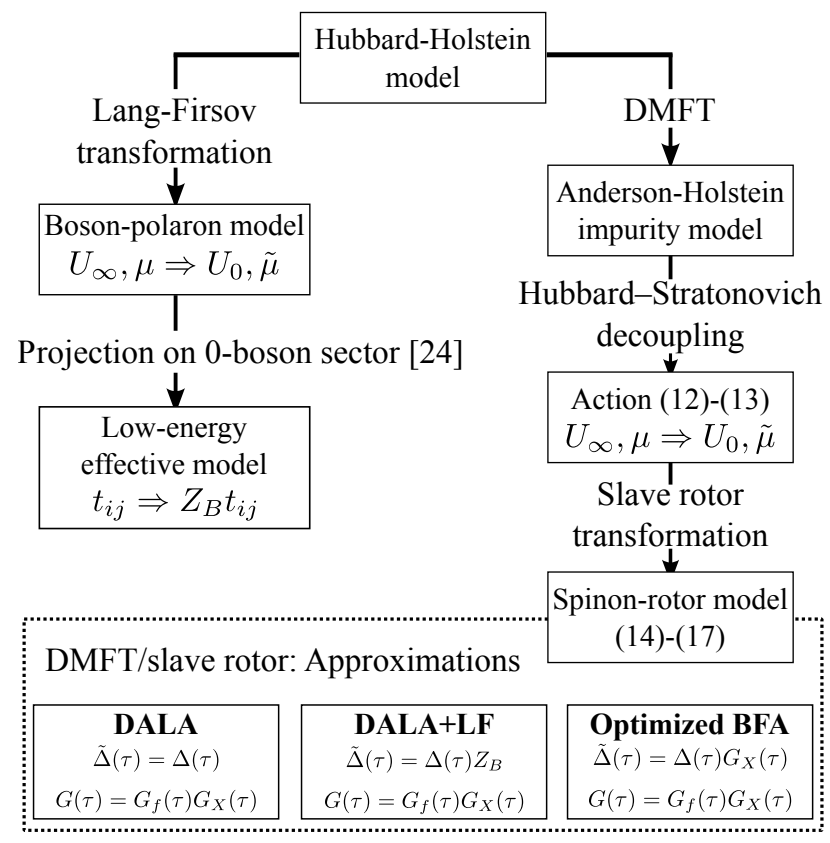

Figure 7: Relations between some models and methods described in the paper.

approximation is closely related to DALA+LF, but does not suffer from spectral weight loss issues. It can also be used together with static- $U$ impurity solvers and allows one to reconstruct spectra with a rich resonance structure.

All main models and methods mentioned in the paper, as well as their relations, are summarized in Fig. 7.

A direct comparison of the methods shows that the results of the optimized BFA are close to those of DALA+LF at low temperatures, but the difference should arguably be larger for higher temperatures, when more boson excitations come into play.

An obvious further development of the proposed method will be a generalization to multiband models. This task seems straightforward, in full analogy to DALA. Another interesting possibility is the calculation of higher correlation functions. Indeed, transformation (7) together with expressions for higher atomic correlators of rotors gives a simple way to calculate such quantities [see (A5) in Appendix A. This could be of special interest in the context of the dual boson approach 4 .

\section{ACKNOWLEDGMENTS}

We would like to thank A. Lichtenstein for interesting and inspiring discussions, and M. Casula for collaborations around the DALA and DALA+LF schemes as implemented in [11. We are grateful to Serge Florens for providing us with his implementation of the slave rotor method. This work was supported by the French ANR under Projects No. SURMOTT and No. PNICTIDES, the European Research Council under Project
No. 617196, as well as by the Deutsche Forschungsgemeinschaft through Project No. SFB668-A3. We acknowledge computing time by IDRIS/GENCI Orsay, under Project No. 091393.

\section{Appendix A: Correlation functions in the atomic limit}

A general $2 n$-point correlation function of slave rotors is defined as the following average value:

$$
G_{X}\left(\tau_{1} \ldots \tau_{n} ; \tau_{1}^{\prime} \ldots \tau_{n}^{\prime}\right)=\left\langle e^{i \theta\left(\tau_{1}\right)-i \theta\left(\tau_{1}^{\prime}\right)+\ldots+i \theta\left(\tau_{n}\right)-i \theta\left(\tau_{n}^{\prime}\right)}\right\rangle
$$

In the atomic limit, the averaging is performed with the action $S_{\mathrm{dyn}}[\theta]$ given by Eq. (17). In spite of the fact that $S_{\text {dyn }}$ is quadratic in the phase variable $\theta(\tau)$, there is no standard Wick's theorem, since $\theta(\tau)$ enters the definition of the correlation function in an exponential form. Nonetheless, the calculation of the higher-order correlators is straightforward.

By definition, we have

$$
\begin{aligned}
& G_{X}^{\mathrm{at}}\left(\tau_{1} \ldots \tau_{n} ; \tau_{1}^{\prime} \ldots \tau_{n}^{\prime}\right)= \\
& \quad=\frac{\int \mathcal{D}[\theta] e^{i \theta\left(\tau_{1}\right)-i \theta\left(\tau_{1}^{\prime}\right)+\ldots+i \theta\left(\tau_{n}\right)-i \theta\left(\tau_{n}^{\prime}\right)-S_{\mathrm{dyn}}[\theta]}}{\int \mathcal{D}[\theta] e^{-S_{\mathrm{dyn}}[\theta]}} .
\end{aligned}
$$

The phase field fluctuating in imaginary time is represented as a sum over all bosonic Matsubara frequencies except $\nu=0$,

$$
\theta(\tau)=\frac{1}{\beta} \sum_{\nu \neq 0} \theta_{\nu} e^{-i \nu \tau} .
$$

The ratio of the integrals in $\mathrm{A} 2$ breaks up into a product over all nonzero frequencies. Since $\theta(\tau)$ is real, its Fourier components obey the condition $\theta_{\nu}=\theta_{-\nu}^{*}$, and independent integration variables in the path integrals above must be amplitudes at positive frequencies only:

$$
\begin{gathered}
G_{X}^{\mathrm{at}}\left(\tau_{1} \ldots \tau_{n} ; \tau_{1}^{\prime} \ldots \tau_{n}^{\prime}\right)=\prod_{\nu>0} I_{\nu}\left(\tau_{1} \ldots \tau_{n} ; \tau_{1}^{\prime} \ldots \tau_{n}^{\prime}\right), \\
I_{\nu}\left(\tau_{1} \ldots \tau_{n} ; \tau_{1}^{\prime} \ldots \tau_{n}^{\prime}\right) \equiv \\
\equiv \frac{\int_{-\infty}^{+\infty} d \theta_{\nu}^{\prime} d \theta_{\nu}^{\prime \prime} e^{\frac{i}{\beta}\left[\theta_{\nu}\left(\sum_{k=0}^{n} e^{-i \nu \tau_{k}}-e^{-i \nu \tau_{k}^{\prime}}\right)+c . c .\right]-\frac{1}{2 \beta} \frac{\nu^{2}\left|\theta_{\nu}\right|^{2}}{U(i \nu)}}}{\int_{-\infty}^{+\infty} d \theta_{\nu}^{\prime} d \theta_{\nu}^{\prime \prime} e^{-\frac{1}{2 \beta} \frac{\nu^{2}\left|\theta_{\nu}\right|^{2}}{\bar{U}(i \nu)}}} .
\end{gathered}
$$

The integrals in A3 are Gaussian and easily doable. The resulting correlation function reads

$$
\begin{aligned}
& G_{X}^{\mathrm{at}}\left(\tau_{1} \ldots \tau_{n} ; \tau_{1}^{\prime} \ldots \tau_{n}^{\prime}\right)= \\
& =\exp \left[-\frac{1}{\beta} \sum_{\nu>0} \frac{\bar{U}(i \nu)}{\nu^{2}}\left|\sum_{k=1}^{n}\left(e^{-i \nu \tau_{k}}-e^{-i \nu \tau_{k}^{\prime}}\right)\right|^{2}\right] .
\end{aligned}
$$


The transformation (7) immediately gives us the correlator of physical electrons through the directly measurable correlator of spinons,

$$
\begin{aligned}
& G\left(\tau_{1} \ldots \tau_{n} ; \tau_{1}^{\prime} \ldots \tau_{n}^{\prime}\right)= \\
& =G_{f}\left(\tau_{1} \ldots \tau_{n} ; \tau_{1}^{\prime} \ldots \tau_{n}^{\prime}\right) G_{X}^{\mathrm{at}}\left(\tau_{1} \ldots \tau_{n} ; \tau_{1}^{\prime} \ldots \tau_{n}^{\prime}\right) .
\end{aligned}
$$

There is a special case for this equation, which is of high practical importance. If all values of $\tau$ are equal to those of $\tau^{\prime}$ (up to a possible index permutation), $G_{X}=1$ by definition. Therefore, averaged quantities such as $\langle N(\tau) N(0)\rangle$ or $\left\langle S_{z}(\tau) S_{z}(0)\right\rangle$ are identical for physical electrons and spinons.

\section{Appendix B: Renormalization factor $Z_{B}$ at finite temperatures}

At finite temperature, states with many bosons may effectively participate in screening. Thus, a reasonable procedure to calculate $Z_{B}$ would be to average the hopping term of $\hat{H}_{\mathrm{LF}}$ over all bosonic states with a Gibbs weight distribution (for simplicity's sake, we focus on the model with a single boson mode of energy $\left.\omega_{0}\right)$ :

$$
\begin{gathered}
Z_{B} t_{i j} d_{i \sigma}^{\dagger} d_{j \sigma} \equiv t_{i j} \operatorname{Tr}\left[c_{i \sigma}^{\dagger} c_{j \sigma} \hat{\rho}_{B}\right] \\
\hat{\rho}_{B}=\frac{\exp \left(-\beta \omega_{0} \sum_{i} b_{i}^{\dagger} b_{i}\right)}{\operatorname{Tr}\left[\exp \left(-\beta \omega_{0} \sum_{i} b_{i}^{\dagger} b_{i}\right)\right]} .
\end{gathered}
$$

The calculation of the trace consists of two steps. In the first step, we calculate the matrix element of $c_{i \sigma}^{\dagger} c_{j \sigma}$ between states with definite numbers of bosons $n_{i}$. The hopping amplitudes $t_{i j}$ are zero for $i=j$, so the matrix element factorizes as follows:

$\left\langle n_{i} n_{j}\left|c_{i \sigma}^{\dagger} c_{j \sigma}\right| n_{i} n_{j}\right\rangle=\left\langle n_{i}\left|\hat{D}\left(\lambda / \omega_{0}\right)\right| n_{i}\right\rangle\left\langle n_{j}\left|\hat{D}\left(-\lambda / \omega_{0}\right)\right| n_{j}\right\rangle$, where $\hat{D}(\gamma)=\exp \left(\gamma b^{\dagger}-\gamma^{*} b\right)$ is a bosonic displacement operator. Using the Baker-Campbell-Hausdorff formula and a finite-sum representation of the Laguerre polynomials $L_{n}$, we find

$$
\begin{aligned}
&\left\langle n\left|\hat{D}\left(-\lambda / \omega_{0}\right)\right| n\right\rangle= \\
&=\exp \left(-\frac{\lambda^{2}}{2 \omega_{0}^{2}}\right) \sum_{k=0}^{n} \frac{1}{k !}\left(-\frac{\lambda^{2}}{\omega_{0}^{2}}\right)^{k}\left(\begin{array}{l}
n \\
k
\end{array}\right)= \\
&=\exp \left(-\frac{\lambda^{2}}{2 \omega_{0}^{2}}\right) L_{n}\left(\frac{\lambda^{2}}{\omega_{0}^{2}}\right) .
\end{aligned}
$$

In the second step of the derivation, we do an actual averaging over a thermal state,

$$
\begin{aligned}
& Z_{B}= \\
& =\left(1-e^{-\beta \omega_{0}}\right)^{2} \exp \left(-\frac{\lambda^{2}}{\omega_{0}^{2}}\right)\left[\sum_{n=0}^{\infty} e^{-\beta \omega_{0} n} L_{n}\left(\frac{\lambda^{2}}{\omega_{0}^{2}}\right)\right]^{2}= \\
& =\exp \left(-\frac{\lambda^{2}}{\omega_{0}^{2}} \operatorname{coth}\left(\beta \omega_{0} / 2\right)\right), \quad(\mathrm{B} 4)
\end{aligned}
$$

or, in the multiple bosons case,

$$
\begin{aligned}
\ln Z_{B}=-\sum_{\alpha} \frac{\lambda_{\alpha}^{2}}{\omega_{\alpha}^{2}} \operatorname{coth}\left(\beta \omega_{\alpha} / 2\right)= \\
\quad=\frac{1}{\pi} \int_{0}^{+\infty} \frac{\Im U_{\text {ret }}(\epsilon)}{\epsilon^{2}} \operatorname{coth}(\beta \epsilon / 2) d \epsilon .
\end{aligned}
$$

This is the result announced in Sec. (V).
1 E. Berger, P. Valášek, W. von der Linden. Twodimensional Hubbard-Holstein model. Phys. Rev. B 52, 4806 (1995)

2 M. Capone, C. Castellani, M. Grilli. Electron-Phonon Interaction in Strongly Correlated Systems. Adv. Cond. Mater. Phys. 2010, 920860 (2010)

${ }^{3}$ L. Proville, S. Aubrya. Small bipolarons in the 2dimensional Holstein-Hubbard model. I. The adiabatic limit. Europhys. J. B 11, 41 (1999)

4 J. Boncă, T. Katrašnik, S. A. Trugman. Mobile Bipolaron. Phys. Rev. Lett. 84, 3153 (2000)

5 J. K. Freericks, M. Jarrell. Competition between electronphonon attraction and weak Coulomb repulsion. Phys. Rev. Lett. 75, 2570 (1995)

6 Y. Takada. Superconductivity in the half-filled HubbardHolstein model in the antiadiabatic region. J. Phys. Soc.
Jpn. 65, 1544 (1996)

7 M. Capone, G. Sangiovanni, C. Castellani, C. Di Castro, M. Grilli. Phase separation close to the density-driven Mott transition in the Hubbard-Holstein model. Phys. Rev. Lett. 92, 106401 (2004)

8 G. Sangiovanni, M. Capone, C. Castellani, M. Grilli. Electron-Phonon interaction close to a Mott Transition. Phys. Rev. Lett. 94, 026401 (2005)

9 C. Castellani, C. Di Castro, M. Grilli. Singular Quasiparticle Scattering in the Proximity of Charge Instabilities. Phys. Rev. Lett. 75, 4650 (1995)

10 Ph. Werner, M. Casula, T. Miyake, F. Aryasetiawan, A. J. Millis, S. Biermann. Satellites and large doping and temperature dependence of electronic properties in hole-doped $\mathrm{BaFe}_{2} \mathrm{As}_{2}$. Nature Physics 8, 331-337 (2012)

11 M. Casula, A. Rubtsov, S. Biermann. Dynamical screen- 
ing effects in correlated materials: Plasmon satellites and spectral weight transfers from a Green's function ansatz to extended dynamical mean field theory. Phys. Rev. B 85, 035115 (2012)

12 V. I. Anisimov, A. I. Poteryaev, M. A. Korotin, A. O. Anokhin, G. Kotliar. First-principles calculations of the electronic structure and spectra of strongly correlated systems: dynamical mean-field theory. J. Phys.: Condens. Matter 9, 7359 (1997)

13 A. I. Lichtenstein, M. I. Katsnelson. Electronic structure calculations with dynamical mean-field theory: $L D A++$ approach. Phys. Rev. B 57, 6884 (1998)

14 V. I. Anisimov, J. Zaanen, O. K. Andersen. Band theory and Mott insulators: Hubbard U instead of Stoner I. Phys. Rev. B 44, 943 (1991)

15 A. Georges, G. Kotliar, W. Krauth and M. J. Rozenberg. Dynamical mean-field theory of strongly correlated fermion systems and the limit of infinite dimensions. Rev. Mod. Phys. 68, 13 (1996)

16 S. Biermann. "LDA+DMFT" - a Tool for Investigating the Electronic Structure of Materials with Strong Electronic Coulomb Correlations. Encyclopedia of Materials: Science and Technology. Edited by J. Buschow (Elsevier, New York, 2005).

17 J. M. Tomczak, A. I. Poteryaev, S. Biermann. Momentumresolved spectroscopy of correlated metals: a view from $d y$ namical mean field theory. C. R. Phys. 10, No. 6537 (2009)

18 J. M. Tomczak, S. Biermann. Optical Properties of Correlated Materials: Generalized Peierls Approach and its Application to $\mathrm{VO}_{2}$. Phys. Rev. B. 80085117 (2009)

19 J. M. Tomczak, S. Biermann. Materials Design using Correlated Oxides: Optical Properties of Vanadium Dioxide. EPL 86, 37004 (2009)

20 J. M. Tomczak, K. Haule, G. Kotliar. Signatures of electronic correlations in iron silicide. Proc. Natl. Acad. Sci. USA 109 (9) 3243-3246 (2012)

21 F. Aryasetiawan, M. Imada, A. Georges, G. Kotliar, S. Biermann, A. I. Lichtenstein. Frequency-dependent local interactions and low-energy effective models from electronic structure calculations. Phys. Rev. B 70, 195104 (2004)

22 T. Miyake and F. Aryasetiawan. Screened Coulomb interaction in the maximally localized Wannier basis. Phys. Rev. B 77, 085122 (2008)

${ }^{23}$ Loïg Vaugier, Hong Jiang, and Silke Biermann. Hubbard $U$ and Hund exchange $J$ in transition metal oxides: Screening versus localization trends from constrained random phase approximation Phys. Rev. B 86, 165105 (2012)

24 M. Casula, Ph. Werner, L. Vaugier, F. Aryasetiawan, T. Miyake, A. J. Millis, S. Biermann. Low-Energy Models for Correlated Materials: Bandwidth Renormalization from Coulombic Screening. Phys. Rev. Lett. 109, 126408 (2012)

25 S. Biermann, F. Aryasetiawan, A. Georges. FirstPrinciples Approach to the Electronic Structure of Strongly Correlated Systems: Combining the GW Approximation and Dynamical Mean-Field Theory. Phys. Rev. Lett. 90, 086402 (2003)

26 P. Sun and G. Kotliar. Many-Body Approximation Scheme beyond GW. Phys. Rev. Lett. 92, 196402 (2004)

27 Q. Si and J. L. Smith, Kosterlitz-Thouless transition and short range spatial correlations in an extended Hubbard model, Phys. Rev. Lett. 77, 3391 (1996). H. Kajueter, Ph.D. thesis, Rutgers University, 1996. A. M. Sengupta and A. Georges, Non-Fermiliquid behavior near a $T=0$ spin-glass transition Phys. Rev. B 52, 10295 (1995).

28 J. M. Tomczak, M. Casula, T. Miyake, F. Aryasetiawan, S. Biermann. Combined $G W$ and dynamical mean-field theory: Dynamical screening effects in transition metal oxides. EPL 100, 67001 (2012)

29 J. M. Tomczak, M. Casula, T. Miyake, S. Biermann. Asymmetric band widening by screened exchange competing with local correlations in $\mathrm{SrVO}_{3}$ : new surprises on an old compound from combined $G W$ and dynamical mean field theory $G W+D M F T$. Phys. Rev. B 90, 165138 (2014).

30 S. Biermann, Dynamical screening effects in correlated electron materials - a progress report on combined many-body perturbation and dynamical mean field theory: ' $G W+D M F T$ ', J. Phys. Condens. Matter 26173202 (2014)

31 Rubtsov, A. N. and Savkin, V. V. and Lichtenstein, A. I. Continuous-time quantum Monte Carlo method for fermions. Phys. Rev. B 72, 035122 (2005)

32 Ph. Werner, A. J. Millis. Dynamical Screening in Correlated Electron Materials. Phys. Rev. Lett. 104, 146401 (2010)

${ }^{33}$ L. Huang, Y. Wang. Dynamical screening in strongly correlated metal $\mathrm{SrVO}_{3}$. EPL 99, 67003 (2012)

34 I. G. Lang, Y. A. Firsov. Kinetic theory of semiconductors with low mobility. Soviet Physics JETP 16, 1301 (1963)

${ }^{35} \mathrm{Ph}$. Werner, A. J. Millis. Efficient Dynamical Mean Field Simulation of the Holstein-Hubbard Model. Phys. Rev. Lett. 99146404 (2007)

36 S. Florens, A. Georges. Quantum impurity solvers using a slave rotor representation. Phys. Rev. B 66, 165111 (2002)

37 S. Florens. Coherence and localization in strongly correlated electrons systems $\mathrm{PhD}$ thesis (in French), University of Paris VI, 2003 (unpublished)

38 S. Florens, A. Georges. Slave-rotor mean-field theories of strongly correlated systems and the Mott transitionin finite dimensions. Phys. Rev. B 70, 035114 (2004)

39 E. Zhao, A. Paramekanti. Self-consistent slave rotor meanfield theory for strongly correlated systems. Phys. Rev. B 76, 195101 (2007)

40 Ki-Seok Kim. Bandwidth-control versus doping-control Mott transition in the Hubbard model. Phys. Rev. B 74, 115122 (2006)

41 Wing-Ho Ko, Patrick A. Lee. Magnetism and Mott transition: A slave-rotor study. Phys. Rev. B 83, 134515 (2011)

42 The TRIQS-based hybridization-expansion matrix solver. http://ipht.cea.fr/triqs/1.1/applications/cthyb_ matrix/

43 A. W. Sandvik. Stochastic method for analytic continuation of quantum Monte Carlo data. Phys. Rev. B 57, 10287 (1998)

44 N. Blümer. Mott-Hubbard Metal-Insulator Transition and Optical Conductivity in High Dimensions. (Shaker, Aachen, 2003).

45 A. N. Rubtsov, M. I. Katsnelson, A. I. Lichtenstein. Dual boson approach to collective excitations in correlated fermionic systems. Ann. Phys. 327, 5, 1320 (2012) 